https://helda.helsinki.fi

Constraints on neutrino density and velocity isocurvature modes from WMAP-9 data

\title{
Savelainen, Matti
}

2013-09-27

Savelainen , M , Väliviita , J , Walia , P , Rusak , S \& Kurki-Suonio , H 2013 , ' Constraints on neutrino density and velocity isocurvature modes from WMAP-9 data ' , Physical Review D :

Particles, Fields, Gravitation and Cosmology , vol. 88 , no. 6 , 063010 . https://doi.org/10.1103/PhysRevD.88.063010

http://hdl.handle.net/10138/234264

https://doi.org/10.1103/PhysRevD.88.063010

other

publishedVersion

Downloaded from Helda, University of Helsinki institutional repository.

This is an electronic reprint of the original article.

This reprint may differ from the original in pagination and typographic detail.

Please cite the original version. 


\title{
Constraints on neutrino density and velocity isocurvature modes from WMAP-9 data
}

\author{
Matti Savelainen, ${ }^{*}$ Jussi Väliviita, Parampreet Walia, Stanislav Rusak, and Hannu Kurki-Suonio \\ Department of Physics and Helsinki Institute of Physics, University of Helsinki, P.O. Box 64, \\ FIN-00014 University of Helsinki, Finland \\ (Received 23 July 2013; published 27 September 2013)
}

\begin{abstract}
We use WMAP-9 and other CMB data to constrain cosmological models where the primordial perturbations have both an adiabatic and a (possibly correlated) neutrino density (NDI), neutrino velocity (NVI), or cold dark matter density (CDI) isocurvature component. For NDI and CDI we use both a phenomenological approach, where primordial perturbations are parametrized in terms of amplitudes at two scales, and a slow-roll two-field inflation approach, where slow-roll parameters are used as primary parameters. For NVI we use only the phenomenological approach, since it is difficult to imagine a connection with inflation. We find that in the NDI and NVI cases larger isocurvature fractions are allowed than in the corresponding models with CDI. For uncorrelated perturbations, the upper limit to the primordial NDI (NVI) fraction is $24 \%(20 \%)$ at $k=0.002 \mathrm{Mpc}^{-1}$ and $28 \%(16 \%)$ at $k=0.01 \mathrm{Mpc}^{-1}$. For maximally correlated (anticorrelated) perturbations, the upper limit to the NDI fraction is $3.0 \%(0.9 \%)$. The nonadiabatic contribution to the CMB temperature variance can be as large as $10 \%(-13 \%)$ for the NDI (NVI) modes. Bayesian model comparison favors the pure adiabatic initial mode over the mixed primordial adiabatic and NDI, NVI, or CDI perturbations. At best, the betting odds for a mixed model (uncorrelated NDI) are 1:3.4 compared to the pure adiabatic model. For the phenomenological generally correlated mixed models the odds are about 1:100, whereas the slow-roll approach leads to 1:13 (NDI) and 1:51 (CDI).
\end{abstract}

DOI: $10.1103 /$ PhysRevD.88.063010

\section{INTRODUCTION}

Cosmological models with cold dark matter isocurvature perturbations (CDI) were extensively studied in light of Wilkinson Microwave Anisotropy Probe (WMAP) 7-year data in [1], both in a phenomenological and a multifield inflationary setup. Now we test within a similar framework what information the 9-year WMAP (WMAP-9) data [2,3] and other, smaller scale, cosmic microwave background (CMB) data [4,5] give on possible deviations from adiabaticity, focusing on the less-studied neutrino density and velocity isocurvature (NDI and NVI) modes. For completeness we also update the analysis of the CDI mode. Recently, the CDI, NDI, and NVI modes were discussed in [6] in light of the Planck data, but only using a phenomenological approach and without simultaneously allowing for the possible tensor perturbation component.

Along with the adiabatic mode, the baryon density isocurvature (BDI), CDI, NDI, and NVI modes are the only regular primordial scalar perturbation modes [7]; others are either decaying modes or singular. The BDI and CDI modes are indistinguishable at the linear level in the CMB (see however $[8,9]$ ), and the results for the CDI mode can easily be interpreted as constraints on the total matter density isocurvature.

We define the different perturbation modes using the five perturbation quantities: (1) the curvature perturbation in the comoving gauge, $\mathcal{R},(2)$ the cold dark matter entropy perturbation

\footnotetext{
*matti.savelainen@helsinki.fi
}

PACS numbers: $98.70 . V c, 98.80 .-\mathrm{k}, 98.80 . \mathrm{Cq}$

$$
S_{c r} \equiv \delta_{c}-\frac{3}{4} \delta_{r}
$$

(3) the baryon entropy perturbation

$$
S_{b r} \equiv \delta_{b}-\frac{3}{4} \delta_{r}
$$

(4) the neutrino entropy perturbation

$$
S_{\nu r} \equiv \frac{3}{4}\left(\delta_{\nu}-\delta_{r}\right)
$$

and (5) the relative neutrino heat flux [10]

$$
q_{\nu r} \equiv \frac{4}{3}\left(v_{\nu}-v_{r}\right)
$$

where the $\delta_{i}$ are density contrasts of the different energy components, the $v_{i}$ are velocity perturbation potentials, and $r$ stands for radiation, i.e., photons $(\gamma)$ and neutrinos $(\nu)$. The pure perturbation modes correspond to four of these five quantities vanishing initially (in the limit where conformal time $\tau \rightarrow 0$ ).

Thus in the adiabatic (or pure CDI, BDI, NDI, NVI) mode, initially only $\mathcal{R}$ (or $\left.S_{c r}, S_{b r}, S_{\nu r}, q_{\nu r}\right) \neq 0 .{ }^{1}$

\footnotetext{
${ }^{1}$ Note that sometimes, for example in [11], these modes are defined in terms of $S_{c \gamma} \equiv \delta_{c}-\frac{3}{4} \delta_{\gamma}, S_{b \gamma}, S_{\nu \gamma}$, and $q_{\nu \gamma}$ instead, which leads to a different definition of the NDI mode, since if $S_{\nu r} \neq 0$, then $S_{c r}$ and $S_{b r}$ can vanish initially, but $S_{c \gamma}=S_{b \gamma} \neq 0$ in the limit $\tau \rightarrow 0$. On the other hand, if $S_{\nu \gamma} \neq 0$, then $S_{c \gamma}$ and $S_{b \gamma}$ can vanish initially, but $S_{c r}=S_{b r} \neq 0$ in the limit $\tau \rightarrow 0$. The neutrino perturbation quantities are related by $S_{\nu r}=\left(1-f_{\nu}\right) S_{\nu \gamma}$ and $q_{\nu r}=\left(1-f_{\nu}\right) q_{\nu \gamma}$, where $f_{\nu} \equiv \rho_{\nu} /\left(\rho_{\gamma}+\rho_{\nu}\right) \approx 0.4$ is the neutrino energy density fraction. CAMB uses the definitions with respect to the total radiation, i.e., $S_{\nu r}$ and $q_{\nu r}[10]$.
} 
There is so far no evidence for a CDI, NDI, nor NVI perturbation component, and CMB temperature anisotropy observations require them to be subdominant compared to the adiabatic component [6,12-26].

We assume a power-law power spectrum for the primordial curvature and isocurvature perturbations and for their correlation. We also allow for tensor perturbations, which are a natural prediction of inflationary models. However, we allow only one isocurvature mode at a time, since the more general cases, where we have a mixture of several isocurvature modes in addition to the adiabatic and tensor modes, are quite intractable with the present data. In addition to the generally correlated perturbations, we study special cases with no correlation or with $\pm 100 \%$ correlation. Assuming spatially flat geometry of the Universe, we perform full parameter scans of this mixed (adiabatic and isocurvature) model, as well as the standard adiabatic $\Lambda \mathrm{CDM}$ model. We present posterior probability densities of the standard cosmological parameters and the extra isocurvature parameters and report the Bayesian evidences for the models. These evidences give the betting odds for the pure adiabatic model against the various mixed (isocurvature) models.

We use two different approaches: (1) a phenomenological approach, where we make no reference to the origin of the primordial perturbations and just determine or constrain their amplitudes from the data, allowing the spectra of the adiabatic, isocurvature, and correlation components to be independent; (2) for the CDI and NDI modes, a slow-roll two-field inflation approach, where we assume the perturbations are generated by quantum fluctuations during two-field inflation, and the spectral indices are determined by the slow-roll parameters at the time the cosmological scales exit the horizon during inflation. This approach forces the spectra to be nearly scale invariant, since we assume the magnitude of the slow-roll parameters to be small.

The first approach is good for detecting nonadiabatic features in the data. If these were found, then a further investigation would be motivated. If not, then we can set an upper limit to the nonadiabaticity of the data. The second approach may give answers to questions directly related to inflation and inflationary potential.

We use the same notation as in [1]. A summary of the symbols can be found in Table I of [1]. In Sec. II we introduce our model and its phenomenological and inflationary parametrizations. In Sec. III we review two scenarios for generating the NDI mode. In Sec. IV we describe the data and sampling method. Sections V and VI are devoted to the results. In Sec. VII we discuss why WMAP-9 leads to tighter constraints than the recent Planck data. The main findings are summarized in Sec. VIII.

\section{THE MODEL}

We assume that the primordial perturbation, presented here in Fourier space, is a superposition of the adiabatic mode [characterized by the comoving curvature perturbation $\mathcal{R}(\mathbf{k})$ ] and an isocurvature mode characterized by $S(\mathbf{k})$, where $S$ is either $S_{c r}, S_{\nu r}$, or $q_{\nu r}$. The power spectrum $\mathcal{P}=\mathcal{P}_{\mathcal{R}}+\mathcal{C}_{\mathcal{R} S}+\mathcal{C}_{S \mathcal{R}}+\mathcal{P}_{S}$ and its components are defined by the expectation value

$$
\begin{aligned}
\left\langle[\mathcal{R}(\mathbf{k})+S(\mathbf{k})]^{*}[\mathcal{R}(\tilde{\mathbf{k}})+S(\tilde{\mathbf{k}})]\right\rangle \\
\equiv(2 \pi)^{3} \delta^{(3)}(\mathbf{k}-\tilde{\mathbf{k}}) \times \frac{2 \pi^{2}}{k^{3}}\left[\mathcal{P}_{\mathcal{R}}(k)+\mathcal{C}_{\mathcal{R} S}(k)\right. \\
\left.\quad+\mathcal{C}_{S \mathcal{R}}(k)+\mathcal{P}_{S}(k)\right] .
\end{aligned}
$$

Following [16,21,24,27-29] we divide $\mathcal{R}(\mathbf{k})$ into an uncorrelated part ("ar") and a part fully correlated with $S$ ("as"), and assume power-law forms for the power spectra:

$$
\mathcal{P}_{\mathcal{R}}(k)=\mathcal{P}_{\text {ar }}(k)+\mathcal{P}_{\text {as }}(k),
$$

where

$$
\mathcal{P}_{\text {ar }}(k)=A_{r 0}^{2}\left(\frac{k}{k_{0}}\right)^{n_{\mathrm{ar}}-1}, \quad \mathcal{P}_{\mathrm{as}}(k)=A_{s 0}^{2}\left(\frac{k}{k_{0}}\right)^{n_{\mathrm{as}}-1} .
$$

Since the spectral indices $n_{\mathrm{ar}}$ and $n_{\mathrm{as}}$ are assumed to be constant (i.e., do not depend on $k$ ), the effective single adiabatic spectral index $n_{\text {ad }}^{\text {eff }}(k) \equiv \frac{d \ln \mathcal{P}_{\mathcal{R}}(k)}{d \ln k}+1$ will depend on the scale (i.e., has running), in particular, if $n_{\mathrm{ar}}$ and $n_{\mathrm{as}}$ greatly differ [28].

For the isocurvature and correlation we have

$$
\begin{gathered}
\mathcal{P}_{S}(k)=B_{0}^{2}\left(\frac{k}{k_{0}}\right)^{n_{\text {iso }}-1} \\
\mathcal{C}_{\mathcal{R} S}(k)=\mathcal{C}_{S \mathcal{R}}(k)=A_{S 0} B_{0}\left(\frac{k}{k_{0}}\right)^{n_{\mathrm{cor}}-1},
\end{gathered}
$$

where

$$
n_{\text {cor }}=\frac{n_{\text {as }}+n_{\text {iso }}}{2} .
$$

We denote the values of power spectra at the scale $k_{i}$ by $A_{r i}^{2} \equiv \mathcal{P}_{\text {ar }}\left(k_{i}\right), A_{s i}^{2} \equiv \mathcal{P}_{\text {as }}\left(k_{i}\right)$, and $B_{i}^{2} \equiv \mathcal{P}_{S}\left(k_{i}\right)$. In the following we choose three reference (i.e., pivot) scales:

$$
\begin{gathered}
k_{1}=0.002 \mathrm{Mpc}^{-1}, \quad k_{0}=0.010 \mathrm{Mpc}^{-1}, \\
k_{2}=0.050 \mathrm{Mpc}^{-1} .
\end{gathered}
$$

We further define the total primordial perturbation power,

$$
A_{i}^{2} \equiv A_{r i}^{2}+A_{s i}^{2}+B_{i}^{2},
$$

the primordial isocurvature fraction,

$$
\alpha_{i} \equiv \frac{B_{i}^{2}}{A_{i}^{2}},
$$

and the ratio of the correlated adiabatic component to the total adiabatic power, 


$$
\gamma_{i} \equiv \operatorname{sign}\left(A_{s i} B_{i}\right) \frac{A_{s i}^{2}}{A_{r i}^{2}+A_{s i}^{2}},
$$

so that

$$
\begin{aligned}
A_{r i}^{2} & =\left(1-\left|\gamma_{i}\right|\right)\left(A_{r i}^{2}+A_{s i}^{2}\right)=\left(1-\left|\gamma_{i}\right|\right)\left(1-\alpha_{i}\right) A_{i}^{2}, \\
A_{s i}^{2} & =\left|\gamma_{i}\right|\left(A_{r i}^{2}+A_{s i}^{2}\right)=\left|\gamma_{i}\right|\left(1-\alpha_{i}\right) A_{i}^{2}, \\
B_{i}^{2} & =\alpha_{i} A_{i}^{2}, \\
A_{s i} B_{i} & =\alpha_{\text {cori } i} A_{i}^{2}=\mathcal{C}_{\mathcal{R} S}\left(k_{i}\right)=\mathcal{C}_{S \mathcal{R}}\left(k_{i}\right) .
\end{aligned}
$$

On the last line we define the relative amplitude of the primordial correlation between the adiabatic and isocurvature perturbations, $\alpha_{\text {cor } i} \equiv \operatorname{sign}\left(\gamma_{i}\right) \sqrt{\alpha_{i}\left(1-\alpha_{i}\right)\left|\gamma_{i}\right|}$.

The total CMB temperature angular power spectrum can be written as

$$
\begin{aligned}
C_{\ell}= & A_{0}^{2}\left[\left(1-\alpha_{0}\right)\left(1-\left|\gamma_{0}\right|\right) \hat{C}_{\ell}^{\mathrm{ar}}+\left(1-\alpha_{0}\right)\left|\gamma_{0}\right| \hat{C}_{\ell}^{\mathrm{as}}\right. \\
& \left.+\alpha_{0} \hat{C}_{\ell}^{\mathrm{iso}}+\alpha_{\mathrm{cor} 0} \hat{C}_{\ell}^{\mathrm{cor}}+\left(1-\alpha_{0}\right) r_{0} \hat{C}_{\ell}^{T}\right] \\
\equiv & C_{\ell}^{\mathrm{ar}}+C_{\ell}^{\mathrm{as}}+C_{\ell}^{\mathrm{iso}}+C_{\ell}^{\mathrm{cor}}+C_{\ell}^{T},
\end{aligned}
$$

where the $\hat{C}_{\ell}$ represent the different contributions to the angular power spectrum that would result from a corresponding primordial spectrum with unit amplitude at the pivot scale $k=k_{0}$ (see [29]). $C_{\ell}^{T}$ comes from the primordial tensor perturbations.

The total nonadiabatic contribution to the CMB temperature variance,

$$
\begin{aligned}
\alpha_{T} & \equiv \frac{\left\langle\left(\delta T^{\text {nonad }}\right)^{2}\right\rangle}{\left\langle\left(\delta T^{\text {total from scalar perturbations } \left.)^{2}\right\rangle}\right.\right.} \\
& =\frac{\sum_{\ell=2}^{2100}(2 \ell+1)\left(C_{\ell}^{\text {iso }}+C_{\ell}^{\text {cor }}\right)}{\sum_{\ell=2}^{2100}(2 \ell+1)\left(C_{\ell}^{\mathrm{ar}}+C_{\ell}^{\mathrm{as}}+C_{\ell}^{\mathrm{iso}}+C_{\ell}^{\mathrm{cor}}\right)},
\end{aligned}
$$

is our pivot-scale free measure of the nonadiabaticity.

Our sign convention for $\mathcal{R}$ and $S$ is such that for NDI and NVI a positive primordial correlation leads to a positive contribution to the final $C_{\ell}$ spectrum; i.e., a positive primordial $\gamma\left(\right.$ or $\mathcal{C}_{\mathcal{R} S}(k)>0$ ) gives $C_{\ell}^{\text {cor }}>0$. In the case of CDI this is true in the Sachs-Wolfe region (at low multipoles), but at higher multipoles $C_{\ell}^{\text {cor }}$ keeps changing its sign as a function of $\ell$, although the primordial correlation does not change its sign as a function of $k$ in our model.

\section{A. Phenomenological parametrization}

The above model has six independent scalar perturbation parameters. In the amplitude parametrization we assign uniform priors to the following primary parameters:

$$
\begin{gathered}
\ln \left(10^{10} A_{1}^{2}\right), \ln \left(10^{10} A_{2}^{2}\right) \in(1,7), \quad \alpha_{1}, \alpha_{2} \in(0,1), \\
\gamma_{1} \in(-1,1), \quad \text { and }\left|\gamma_{2}\right| \in(0,1) .
\end{gathered}
$$

As we assume power-law spectra, the sign of correlation cannot be a function of $k$. Therefore, the sign of $\gamma_{2}$ has to be the same as that of $\gamma_{1}$. The background is described by the usual four $\Lambda \mathrm{CDM}$ background parameters, i.e., the physical baryon density, the physical CDM density, the sound horizon angle at last scattering $\theta$, and the optical depth:

$$
\begin{aligned}
\omega_{b} & \equiv \Omega_{b} h^{2} \in(0.01,0.05), \\
\omega_{c} & \equiv \Omega_{c} h^{2} \in(0.02,0.30), \\
100 \theta & \in(0.5,2.2), \\
\tau & \in(0.02,0.30) .
\end{aligned}
$$

In addition, we include in the analysis the primordial tensor perturbations with a power-law power spectrum

$$
\mathcal{P}_{T}(k)=\mathcal{P}_{T}\left(k_{0}\right)\left(\frac{k}{k_{0}}\right)^{n_{T}} .
$$

Their amplitude has been traditionally parametrized by the tensor-to-scalar ratio $r(k) \equiv \mathcal{P}_{T}(k) / \mathcal{P}_{\mathcal{R}}(k)$. In principle, in the phenomenological treatment the tensor perturbations would add two extra parameters, $r_{0}$ and the tensor spectral index $n_{T}$. However, as no tensor nor isocurvature perturbations have been detected so far, allowing $n_{T}$ to be a free parameter would give us too many poorly constrained parameters to make this study feasible. As the isocurvature perturbations are the focus of this paper, we assume even in the phenomenological approach the first inflationary consistency relation, which fixes $n_{T}$; see, e.g., [21,30-33]. Therefore the tensor perturbations add only one extra free parameter, while the tensor spectral index is a derived parameter given by the consistency relation $n_{T 0}=$ $-r_{0} /\left[8\left(1-\left|\gamma_{0}\right|\right)\right]$. We impose also the second consistency relation [33,34], which gives the running $q_{T 0} \equiv d n_{T}$ / $\left.d \ln k\right|_{k=k_{0}}=n_{T 0}\left[n_{T 0}-\left(n_{\mathrm{ar}}-1\right)\right]$.

Assigning a uniform prior for $r_{0}$ and $\gamma_{1,2}$ and using the first inflationary consistency relation, as described above, would lead to an unphysical prior on $n_{T}$. Namely, the tensor spectral index would receive huge negative values whenever $\left|\gamma_{0}\right|$ is near 1 . This is against the very motivation of using the consistency relation, which is to force the tensor spectrum to follow the typical inflationary prediction of near scale invariance, $\left|n_{T}\right| \ll 1$. In addition, the large scale (low multipole) CMB data will not allow for such a huge tensor contribution. Hence, the use of the first consistency relation would artificially exclude any models where $|\gamma|$ is near 1 . (We will demonstrate this later in the end of Sec. VA.) In order to avoid these problems, we will not parametrize the tensor power by $r_{0}$, but instead by

$$
\tilde{r}_{0} \equiv \frac{\mathcal{P}_{T}\left(k_{0}\right)}{\mathcal{P}_{\text {ar }}\left(k_{0}\right)}=\frac{r_{0}}{1-\left|\gamma_{0}\right|}
$$

for which we assign a uniform prior between 0 and 1.35 . When connecting the model to inflation, $\tilde{r}$ is the ratio of tensor to curvature perturbations generated at horizon exit, to the leading order in slow-roll parameters. So it reflects directly the inflationary physics, and hence a uniform prior 
on it is physically motivated. On the other hand, $r$ is a parameter directly related to observables. With our new definition the first consistency relation reads

$$
n_{T 0}=-\frac{\tilde{r}_{0}}{8},
$$

which leads to a uniform prior on the derived parameter $n_{T 0}$ between -0.17 and 0 without being affected by the correlation parameter $\gamma$.

Furthermore, our new $\tilde{r}$ parametrization has an advantage when studying the fully (anti)correlated models, $\gamma= \pm 1$. In these special cases there is no tensor contribution. So, we can turn off tensors in CAMB and set the tensor parameters to constant values, $r=0$ and $n_{T}=0$. However, in the "old parametrization" we cannot recover these special cases from the general case by taking the limit $|\gamma| \rightarrow 1$ and $r_{0} \rightarrow 0$, since this would correspond to $n_{T}=0 / 0$. In the new parametrization the $|\gamma| \rightarrow 1$ tensorless limit naturally gives $r_{0}=\left(1-\left|\gamma_{0}\right|\right) \tilde{r}_{0}=0 \times 0=0$ and $n_{T}=0$. So, although we treat the special cases as separate MULTINEST runs, we can also see the behavior of the posterior in the limit $|\gamma| \rightarrow 1$ from our generally correlated runs.

\section{B. Inflationary slow-roll parametrization}

In the inflationary slow-roll approach we assume that during inflation there exists at least two "active" fields. The field space can be locally rotated so that the perturbations can be described by an adiabatic component, which is a perturbation in the direction of the background trajectory $\sigma$, and an "isocurvature" component, which is a perturbation in the perpendicular direction $s$. Now we can define four slow-roll parameters that are calculated from the inflationary potential $V(\sigma, s)$ at the time the interesting scale exits the horizon as follows:

$$
\begin{aligned}
\eta_{\sigma \sigma} & =\frac{1}{8 \pi G} \frac{\partial_{\sigma} \partial_{\sigma} V}{V}, \\
\eta_{\sigma s} & =\frac{1}{8 \pi G} \frac{\partial_{\sigma} \partial_{s} V}{V}, \\
\eta_{s s} & =\frac{1}{8 \pi G} \frac{\partial_{s} \partial_{s} V}{V}, \\
\varepsilon & =\frac{1}{16 \pi G}\left(\frac{\partial_{\sigma} V}{V}\right)^{2} .
\end{aligned}
$$

From the slow-roll parameters we can determine the spectral indices and the tensor-to-scalar ratio at horizon exit, $\tilde{r}$ :

$$
\begin{aligned}
n_{\mathrm{ar}} & =1-6 \varepsilon+2 \eta_{\sigma \sigma}, \\
n_{\mathrm{as}} & =1-2 \varepsilon+2 \eta_{s s}-4 \eta_{\sigma s} \tan \Delta, \\
n_{\mathrm{iso}} & =1-2 \varepsilon+2 \eta_{s s}, \quad \tilde{r}=16 \varepsilon, \quad n_{T}=-2 \varepsilon,
\end{aligned}
$$

where the primordial correlation angle $\Delta$ is defined by

$$
\cos \Delta \equiv \frac{\mathcal{C}_{\mathcal{R} S}}{\mathcal{P}_{\mathcal{R}}^{1 / 2} \mathcal{P}_{S}^{1 / 2}}=\operatorname{sign}(\gamma) \sqrt{|\gamma|},
$$

with $0 \leq \Delta \leq \pi$. The relations (24) are valid to first order in slow-roll parameters; see [31,32,35-37] and note that, e.g., in [35] $n=0$ stands for a scale-invariant spectrum whereas we have added the conventional 1 (except for tensors), and we use a different sign convention for $\cos \Delta$ and thus also for $\tan \Delta=\operatorname{sign}(\gamma) \sqrt{1-|\gamma|} / \sqrt{|\gamma|}$.

In the slow-roll approach we have the same background parameters as in the phenomenological approach, Eq. (19), but the perturbations are parametrized by the four slow-roll parameters (three $\eta_{i j}$ whose prior is uniform from -0.075 to +0.075 and $\varepsilon$ with the prior range from 0 to 0.075 ), $\gamma_{0} \in(-1,1)$ and $\ln \left(10^{10} A_{0}^{2}\right) \in(1,7)$. Note that unlike in the fully numerical treatments (see, e.g., [38]), we can only allow small magnitudes for the slow-roll parameters; thus, Eq. (24) is accurate enough. Our choice of prior ranges should guarantee that the second order corrections to $n_{\text {ar,iso }}-1$ are less than $\mathcal{O}(10 \%)$.

\section{MECHANISMS THAT MAY PRODUCE NEUTRINO ISOCURVATURE}

There are various mechanisms that can produce (correlated) isocurvature and adiabatic perturbations; see, e.g., Refs. [31,32,35,36,39-75]. The common ingredient of these models is that at least 1 extra degree of freedom is needed in addition to the 1 degree of freedom provided by the single-field slow-roll inflation, which can give rise to only the adiabatic mode. Multifield inflationary models and curvaton/spectator field models are natural candidates for generating primordial CDI or NDI perturbations. In [1] we reviewed several such scenarios, focusing on the CDI case. Here we will provide examples of stimulating the NDI mode.

It has been suggested [76] (see also [77-79]) that NDI might be generated from inhomogeneous lepton asymmetry in the context of the curvaton scenario. In the curvaton scenario the light curvaton field $\chi$ remains subdominant during inflation but may become important once the inflaton has decayed into radiation. This happens because once the Hubble parameter becomes smaller than the mass of the curvaton, the curvaton starts oscillating and behaves like dust and thus loses energy slower than the radiation fluid. This causes the perturbations in the curvaton to be transferred to the curvature perturbation,

$$
\mathcal{R} \simeq \frac{2 R}{3} \frac{\delta \chi_{*}}{\chi_{*}}
$$

where $R \equiv\left(\frac{3 \rho_{\chi}}{4 \rho_{r}+3 \rho_{\chi}}\right)$ dec is evaluated at the time of curvaton decay. If the lepton number is generated before curvaton decay, the NDI perturbation is 


$$
S_{\nu r} \simeq-\lambda \mathcal{R}, \quad \text { with } \quad \lambda=\frac{135}{7}\left(1-f_{\nu}\right)\left(\frac{\xi}{\pi}\right)^{2},
$$

where $\xi \equiv \mu / T$ is the neutrino asymmetry parameter. ${ }^{2}$ Perturbations are fully anticorrelated, and the isocurvature fraction is

$$
\alpha=\frac{\lambda^{2}}{1+\lambda^{2}} .
$$

BBN constrains $|\xi|<0.07$ [80], implying $\alpha \lesssim 10^{-4}$, which is too small to be observed.

If the lepton number is created directly from curvaton decay, however, the neutrino isocurvature perturbation is [76]

$$
S_{\nu r} \simeq \lambda\left(\frac{1-R}{R}\right) \mathcal{R},
$$

and the perturbations are fully correlated with the isocurvature fraction

$$
\alpha=\frac{\lambda^{2}(1-R)^{2}}{R^{2}+\lambda^{2}(1-R)^{2}} .
$$

Now the isocurvature fraction can be significant if the curvaton decays sufficiently early. However, nonGaussianity in the curvaton scenario is

$$
f_{\mathrm{NL}}^{\text {local }}=\frac{5}{4 R}-\frac{5}{3}-\frac{5 R}{6},
$$

which implies $R>0.078$ from the latest Planck $2 \sigma$ constraint $f_{\mathrm{NL}}^{\text {local }}<14.3$ [81]. Equation (31) gives the largest $\alpha$ when $\lambda^{2}$ is the largest possible. Hence, saturating $\lambda$ with the BBN constraint, the above result for $R$ leads to $\alpha<0.0045$.

The NVI mode is more difficult to motivate theoretically because it would have to be generated after neutrino decoupling, and there are no proposed theoretical models to date. Thus we study the NVI mode only in the phenomenological setup.

\section{DATA AND SAMPLING METHOD}

We employ the CMB temperature and polarization anisotropy data: WMAP-9 data [2], the Arcminute Cosmology Bolometer Array Receiver (ACBAR) data [4], and QUEST at DASI (QUaD) data [5] (QUEST stands

\footnotetext{
${ }^{2}$ The nonzero chemical potential $\mu$ of neutrinos affects the effective number of neutrino species by

$$
N_{\nu}^{\text {eff }} \rightarrow \tilde{N}_{\nu}^{\text {eff }} \simeq\left[1+\frac{30}{7}\left(\frac{\xi}{\pi}\right)^{2}+\frac{15}{7}\left(\frac{\xi}{\pi}\right)^{4}\right] N_{\nu}^{\text {eff }} .
$$

Assuming the standard $N_{\nu}^{\text {eff }}=3.046$ and the big bang nucleosynthesis $(\mathrm{BBN})$ constraint $|\xi|<0.07$, we find that the corrected number would be $\tilde{N}_{\nu}^{\text {eff }}=3.052$. Even $|\xi|$ as large as 0.2 would lead to quite a small correction, $\tilde{N}_{\nu}^{\text {eff }}=3.099$. Anyway, we checked that our NDI $\gamma=-1$ runs (see Sec. VIB) lead to virtually identical results with $N_{\nu}^{\text {eff }}=3.046$ and 3.100. So, we can safely perform the analysis with the standard $N_{\nu}^{\text {eff }}=3.046$.
}

for Q and U Extragalactic Survey Telescope, and DASI for Degree Angular Scale Interferometer). The additional CMB data sets are the same as in [1] to make the comparison clear and in order to see whether there are significant differences between WMAP-7 and WMAP-9.

We sample the parameter space using the MULTINEST nested sampling package [82,83]; see also [84-87]. It is easy to interface with CAMB/COSMOMC codes $[88,89]$ that we have modified to handle arbitrarily correlated mixtures of adiabatic and isocurvature perturbations.

\section{RESULTS FOR THE GENERALLY CORRELATED MIXED ADIABATIC AND ISOCURVATURE MODELS}

In this section we let $\gamma_{1,2}$ in the amplitude parametrization or $\gamma_{0}$ and $\eta_{\sigma s}$ in the slow-roll parametrization be free parameters, thus allowing for a general scale-dependent correlation amplitude between the adiabatic and one isocurvature mode (either CDI, NDI, or NVI). These models have four extra parameters compared to the "standard" adiabatic $\Lambda \mathrm{CDM}$ model.

\section{A. Phenomenological approach-Amplitude parametrization for CDI, NDI, and NVI}

The marginalized $1 \mathrm{~d}$ posterior probability density functions (pdf) in the mixed adiabatic and isocurvature models (NDI, NVI, CDI) are compared to the pure adiabatic model in Fig. 1 (primary parameters) and Fig. 2 (selected derived
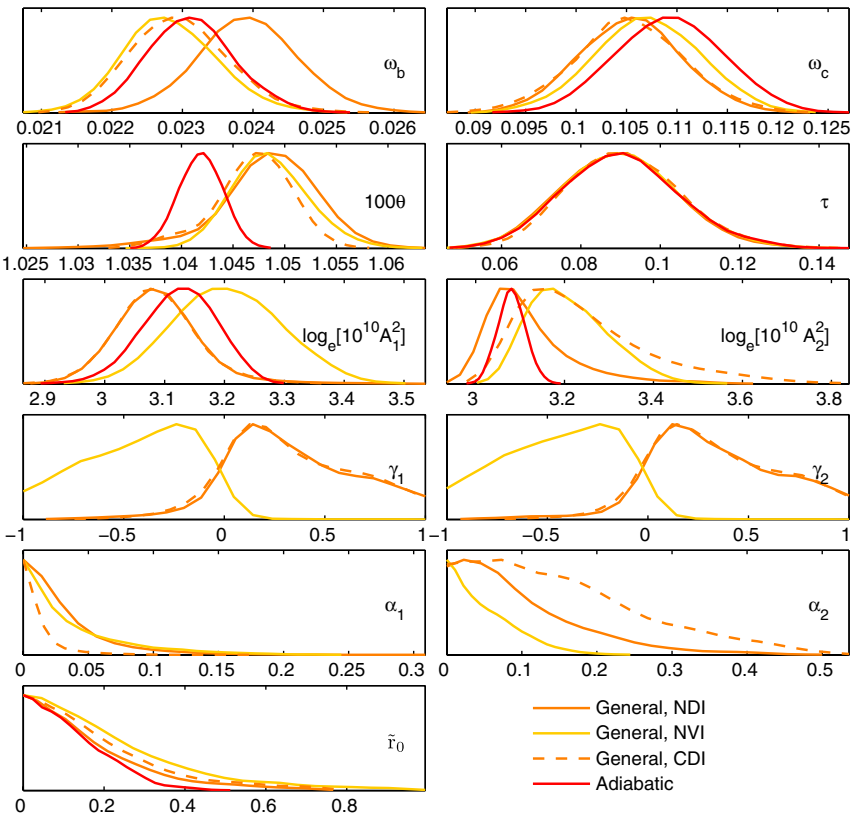

FIG. 1 (color online). Amplitude parametrization, general correlation, primary parameters. Marginalized 1d posterior pdfs of the primary parameters of models with a generally correlated mixture of primordial adiabatic and NDI, NVI, or CDI modes compared to the pure adiabatic model (red/black line). 


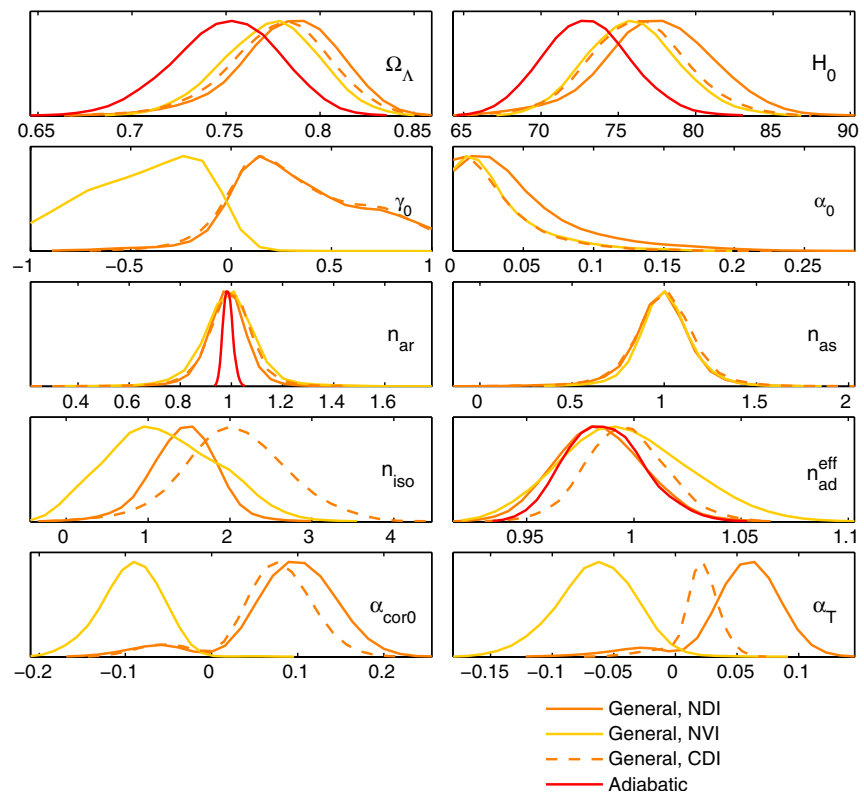

FIG. 2 (color online). Amplitude parametrization, general correlation, derived parameters. Marginalized 1d posterior pdfs as in Fig. 1, but for selected derived parameters.

parameters). In the Appendix we tabulate $68 \%$ or $95 \%$ confidence level (C.L.) intervals for selected parameters and the Bayesian evidence $-\log Z$ (see the third column of Tables II, III, and IV for the generally correlated mixed models).

For the NDI and CDI modes a positive correlation ${ }^{3}$ with the curvature perturbations is preferred by the data, whereas in the case of the NVI mode, a negative correlation is preferred. This can be explained by the temperature angular power spectra of Fig. 3. In the left panel we plot the angular power spectra resulting from pure isocurvature or pure adiabatic scale-invariant primordial perturbations with the same background parameters. The CDI and NDI modes produce an acoustic peak that is to the right of the first acoustic peak of the adiabatic case, whereas the NVI mode leads to a peak that is slightly to the left. For a long time it has been known that in the CDI case the WMAP data prefer minimizing the CDI contribution everywhere $[1,16,24,29]$. Since there is $\ell^{-2}$ damping of the CDI mode compared to the adiabatic mode (see again the left panel of Fig. 3), the overall minimization of the isocurvature contribution is achieved by a relatively large $n_{\text {iso }}$. With WMAP-9 and other CMB data used in this paper, the median of the posterior pdf for CDI is $n_{\text {iso }}=2.05$ (Fig. 2 and Table IV).

In the middle (right) panel of Fig. 3 we show a typical well-fitting adiabatic model and the isocurvature (nonadiabatic, i.e., isocurvature + correlation) contributions of the well-fitting mixed adiabatic and isocurvature models.

\footnotetext{
${ }^{3}$ Recall our sign convention presented after Eq. (17).
}

For these plots we used the median values of the 1d pdf of each parameter. So, these $C_{\ell}$ curves are "representative" of the curves in the good-fit region. Compared to the correlation contribution, the actual isocurvature contribution is negligible. Therefore, we focus on the right panel. For the CDI mode, as well as for the NDI mode, the correlation component would push the first acoustic peak toward the right, compared to the pure adiabatic case, which fits the data very well. To push the peak back toward the left (in order to fit the data) we need, in these models, a larger sound horizon angle; see the solid orange/gray (NDI) and dashed orange/gray (CDI) pdfs for $100 \theta$ in Fig. 1. As explained, e.g., in $[1,16,24,29]$ this leads to a larger Hubble parameter $H_{0}$, a larger $\Omega_{\Lambda}$, and a smaller $\omega_{c}$ than in the pure adiabatic model, since we are studying models with a flat spatial geometry $\left(\Omega_{\text {tot }}=1\right)$.

The phase of the NVI mode is very different from the NDI and CDI. Positively correlated NVI would tend to add power to the left side of the first adiabatic acoustic peak and to reduce the relative power on the right side of the peak. In the mixed model this would move the first acoustic peak to the left compared to the pure adiabatic model. However, a negative NVI correlation works in the opposite way, and hence leads to a very similar effect as the positive correlation in the NDI and CDI cases. Then the pdfs of the primary background parameters $\omega_{c}, \theta$, and $\tau$ (Fig. 1), as well as the derived parameters $\Omega_{\Lambda}$ and $H_{0}$ (Fig. 2), are very similar in all the mixed models, whereas the pdfs of $\gamma_{1,2,0}$ for the mixed NVI model are rough mirror images of those of the mixed NDI and CDI models.

The constraints on the primordial isocurvature fraction are tightest for the CDI on large scales (see $\alpha_{1}$ in Fig. 1) and weakest on small scales (see $\alpha_{2}$ in Fig. 1). This is reflected in the derived parameter $n_{\text {iso }}$ in Fig. 2. Indeed, assuming that the data do not like any isocurvature contribution, the preferred order of the values of $n_{\text {iso }}$ could have been guessed by seeing the left panel of Fig. 3. A nearly scale-invariant spectrum is preferred in the NVI case, since this type of NVI mode leads to a roughly constant fractional isocurvature contribution over the whole range of acoustic peaks.

The primordial correlation amplitude $\alpha_{\text {cor }}$ describes best the primordial deviation from pure adiabaticity, unless the correlation parameter $\gamma$ is (nearly) zero. We find at $k_{0}=$ $0.01 \mathrm{Mpc}^{-1}$ a constraint $-0.08<\alpha_{\text {cor } 0}<0.18$ for NDI, $-0.16<\alpha_{\text {cor } 0}<-0.03$ for NVI, and $-0.08<\alpha_{\text {cor } 0}<$ 0.15 for CDI at $95 \%$ C.L. For the primordial isocurvature fraction the corresponding numbers are $\alpha_{0}<0.14$ (NDI), 0.10 (NVI), and 0.10 (CDI). The CMB data do not show any preference for the mixed models: all the posterior pdfs of the primordial isocurvature fraction $\alpha$ peak at zero or very near zero. The improvement of the $\chi^{2}$ of the best-fitting models compared to the adiabatic model does not exceed the number of extra parameters introduced by the isocurvature modes. 

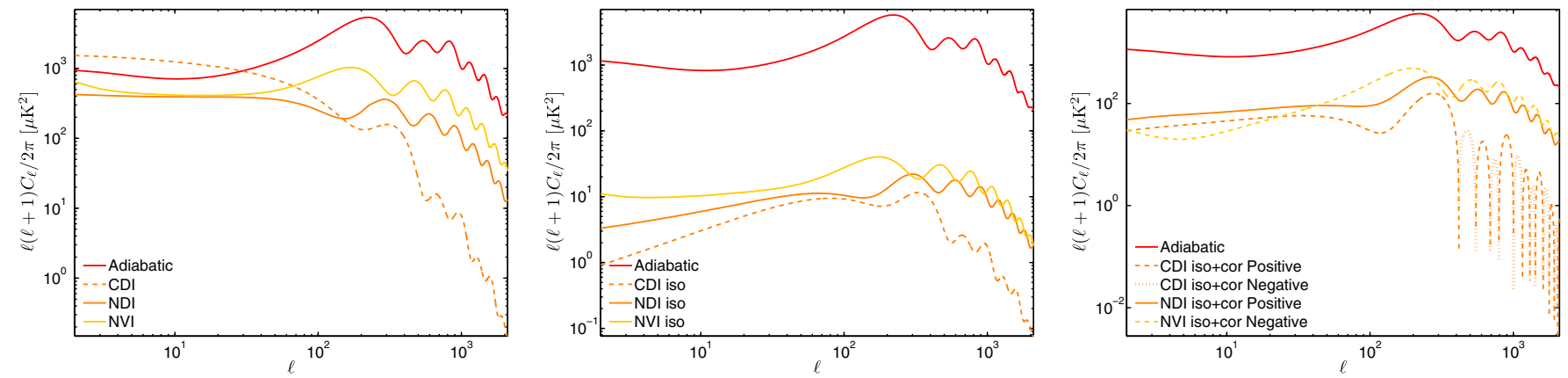

FIG. 3 (color online). CMB temperature angular power spectra. The left panel shows the angular power spectrum resulting from scale-invariant primordial pure CDI, NDI, or NVI perturbations with $\mathcal{P}_{S}=2.4 \times 10^{-9}$, and the pure adiabatic spectrum (red/black) with $\mathcal{P}_{\mathcal{R}}=2.4 \times 10^{-9}$. The middle panel shows a typical well-fitting adiabatic model, and the isocurvature contributions in the typical well-fitting mixed models (correlated adiabatic and CDI, NDI, or NVI primordial perturbations), found in the amplitude parametrization. The right panel is the same as the middle panel, except instead of the isocurvature contribution it shows the total nonadiabatic contribution, i.e., the sum of isocurvature and correlation components.

From Fig. 2 we see that a larger nonadiabatic contribution $\alpha_{T}$ to the CMB temperature variance is allowed by the data in the case of the neutrino isocurvature modes than for the CDI mode. This is because the $C_{\ell}$ contribution from the neutrino modes is not as much off-phase from the adiabatic contribution (and the data agree well with this adiabatic placement of the acoustic peaks); see Fig. 3 . Another contributor to this result is that in the CDI case the correlation component $C_{\ell}^{\text {cor }}$ keeps changing its sign as a function of the multipole, whereas in the neutrino isocurvature cases $C_{\ell}^{\text {cor }}$ has the same sign as the primordial correlation over the whole multipole range. Thus, in the CDI case, there are some cancellations in the summation over $\ell$ in Eq. (17). This may lead to a smaller nonadiabatic contribution to the total $\mathrm{CMB}$ temperature variance than to the individual multipoles in the CDI case. The $95 \%$ C.L. constraints are $-5 \%<\alpha_{T}<10 \%$ (NDI), $-13 \%<\alpha_{T}<-1 \%$ (NVI), and $-3 \%<\alpha_{T}<5 \%$ (CDI). The missing of the adiabatic models $\left(\alpha_{T}=0\right)$ from the $95 \%$ C.L. interval of the NVI case is due to the very similar acoustic peak structures of the NVI and adiabatic modes. Thus the NVI mode is the most difficult to distinguish from the adiabatic one. However, as written above, the pdf of the primordial isocurvature fraction peaks at zero even in the NVI case.

Now we comment on the Bayesian evidences reported on the last lines of Tables II, III, and IV. The adiabatic model is favored. It has $-\ln Z \approx 3,901.17$, whereas the mixed models (with general correlation) all have $-\ln Z \approx$ $3,905 \ldots 3,906$. So the betting odds in favor of the pure adiabatic model against the mixed models are roughly 100:1.

Finally, in Fig. 4 we show the effect of our new $\tilde{r}$ parametrization on the posterior pdfs of $\gamma_{1,2,0}$ and $r_{0}$ (which in the $\tilde{r}$ parametrization is a derived parameter). The constraints of $r_{0}$ are not much affected, but as we expected, in $\tilde{r}$ parametrization the values of $\gamma$ are constrained by the data, not by the unphysical prior of the derived parameter $n_{T}$ (tensor spectral index). As a result our new constraints on $\gamma$ are weaker than those presented in [1] for the CDI case with WMAP-7 or those presented in [21] for NDI, NVI, and CDI cases with WMAP-3 data. Naturally, the posterior pdfs of parameters other than $\gamma$ or $r$ are also affected to some extent. For example, we obtain
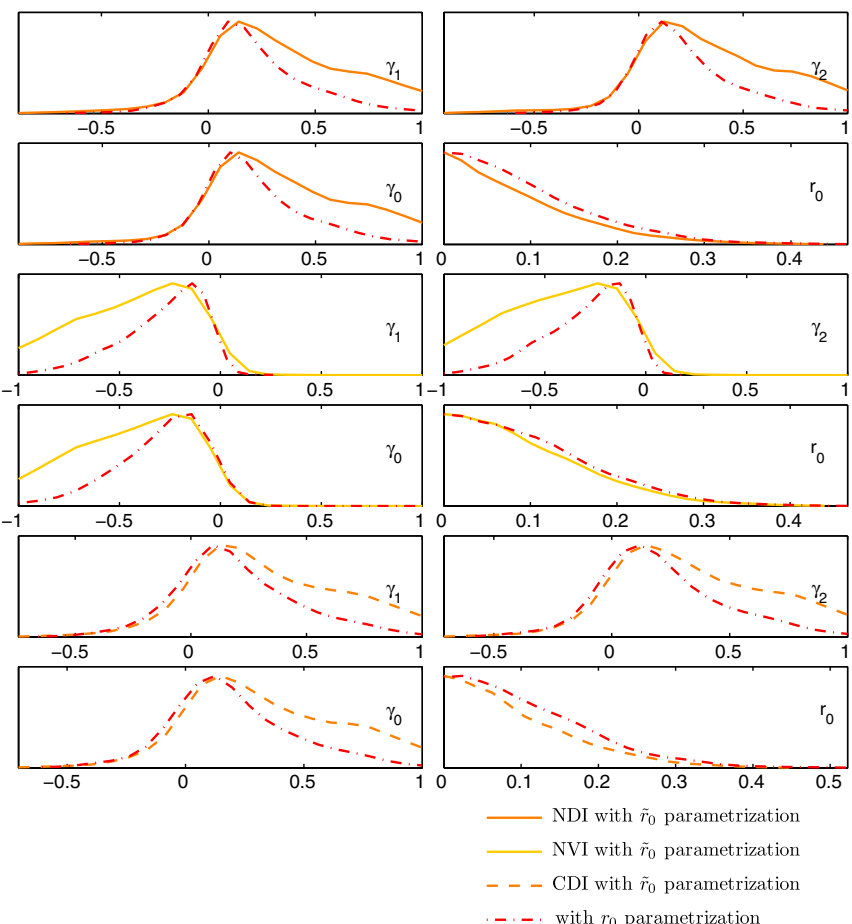

FIG. 4 (color online). Amplitude parametrization, comparison of $\tilde{r}$ and $r$ tensor-to-scalar ratio parametrization. Marginalized $1 \mathrm{~d}$ posterior pdfs of $\gamma_{1}, \gamma_{2}, \gamma_{0}$, and the tensor-to-scalar ratio $r_{0}$ (the ratio of the tensor perturbation power to total curvature perturbation power at primordial time) in the mixed NDI (top four panels), NVI (middle four panels), and CDI (bottom four panels) models with $\tilde{r}_{0}$ (the ratio of the tensor perturbation power to curvature perturbation power at horizon exit during inflation) as a primary parameter, with a uniform prior on it. The dot-dashed (red/black) lines are with $r_{0}$ as a primary parameter. 
slightly tighter constraints on the isocurvature fraction in the new parametrization (since larger correlation fractions are allowed and hence a fixed $\alpha$ leads to a larger nonadiabatic modification).

\section{B. Two-field inflation approach-Slow-roll parametrization for NDI and CDI}

Figure 5 shows marginalized 1d posterior pdfs of the primary parameters in the slow-roll parametrization, and Fig. 6 the selected derived parameters. The medians of the pdfs and $68 \%$ or $95 \%$ C.L. intervals are provided in the Appendix in Tables V and VI for the mixed NDI and CDI models. (As discussed earlier, the NVI mode is hard to think of as resulting from inflationary physics; hence we do not include it in our slow-roll analysis.)

The most significant difference from the previous subsection is that the slow-roll parametrization forces the power spectra, in particular the isocurvature and correlation spectra, to be nearly scale invariant; see Eq. (24). In the CDI case the difference is most dramatic, since the phenomenological approach led to the median $n_{\text {iso }} \sim 2.05$. For NDI the difference is smaller, since it gave the median $n_{\text {iso }} \sim 1.45$. For NVI the slow-roll and phenomenological $\left(n_{\text {iso }} \sim 1.15\right)$ approaches would be almost identical. In the slow-roll parametrization the CDI and NDI modes can significantly modify only the low- $\ell$ part of the $C_{\ell}$ spectrum; see the left panel of Fig. 3. We find tighter constraints than in the phenomenological approach: now $-0.04<\alpha_{\text {cor0 }}<0.15, \alpha_{0}<0.06$ (NDI), and $-0.08<$ $\alpha_{\text {cor0 }}<0.10, \alpha_{0}<0.03(\mathrm{CDI})$.
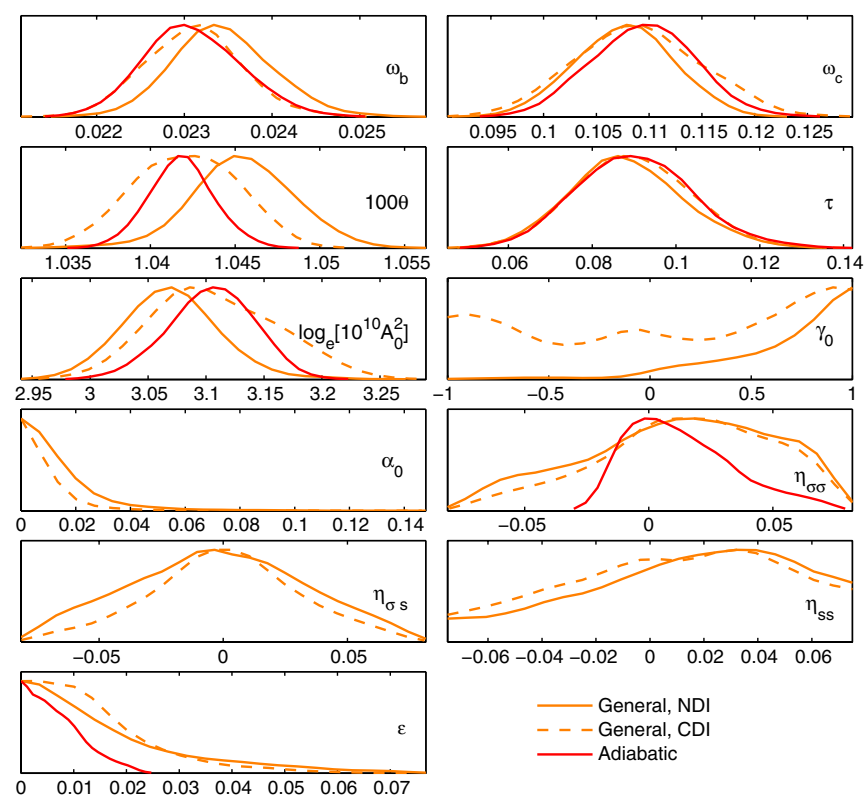

FIG. 5 (color online). Slow-roll parametrization, general correlation, primary parameters. Marginalized 1d posterior pdfs of the primary parameters of models with a generally correlated mixture of primordial adiabatic and NDI or CDI modes compared to the pure adiabatic model (red/black).

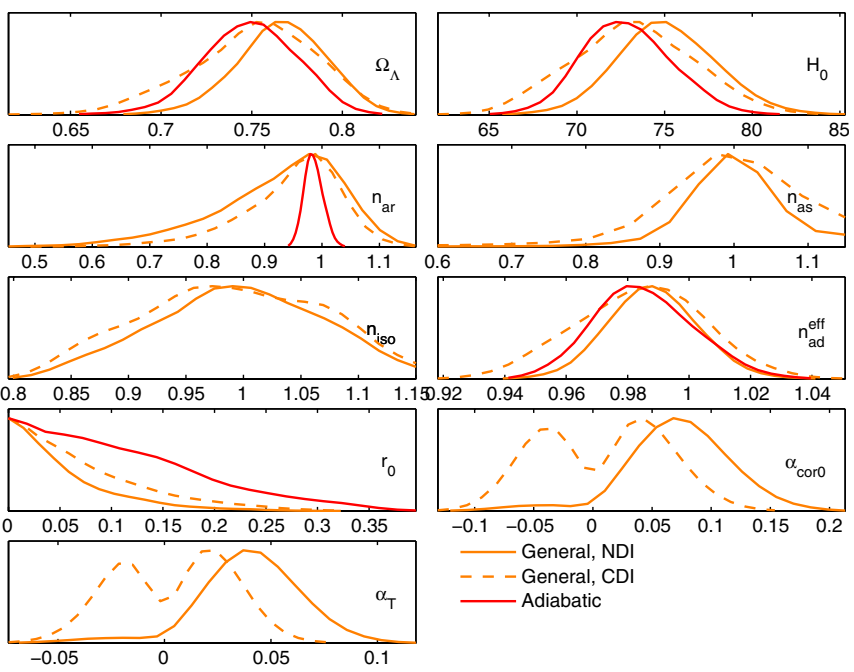

FIG. 6 (color online). Slow-roll parametrization, general correlation, derived parameters. Marginalized 1d posterior pdfs as in Fig. 5, but for selected derived parameters.

A comparison of the posterior pdfs in amplitude and slow-roll parametrization for the NDI case is shown in Fig. 7. (A similar comparison for the CDI case can be found in [1] with WMAP-7 data, or with WMAP-9 by comparing Figs. 1 and 2 to Figs. 5 and 6.) In both the NDI and CDI cases, the preferred values of most of the parameters in the slow-roll parametrization are between preferred values of the pure adiabatic case and the amplitude parametrization. The reason for this is that the near scale invariance of the primordial isocurvature spectrum prevents any significant nonadiabatic contribution to the acoustic peak structure, thus leaving the high- $\ell$ part of the angular power spectrum virtually "adiabatic."

The data prefer positive correlation between the NDI and adiabatic modes, as happens also in the phenomenological case. The nearly scale-invariant NDI mode is able to modify the first acoustic peak almost in the same way as in the amplitude parametrization (with $n_{\text {iso }} \sim 1.45$ ) if the correlation fraction is large enough (compare the left and right panels of Fig. 3). Thus, in the slow-roll parametrization much larger correlation fractions are favored; the pdf of $\gamma_{0}$ peaks at 1 -at the full correlation. The situation is very different for the CDI mode; see $\gamma_{0}$ in Fig. 5. While positive correlation was clearly preferred in the amplitude parametrization (due to the effects on the first acoustic peak), now any correlation fraction $\gamma_{0}$ between -1 and +1 is allowed. As the only effect of the correlated CDI in the slow-roll parametrization is to add or reduce some power at low $\ell$, which is dominated by cosmic variance, the data are insensitive to the sign of the correlation. Thus, for example, the parameters $\alpha_{\text {coro } 0}$ and $\alpha_{T}$ just reflect the uncertainty caused by the cosmic variance, and their pdf is almost symmetric about zero.

In particular, in the NDI case, the tensor-to-scalar ratio $r_{0}$ is constrained more tightly in the slow-roll parametrization, 


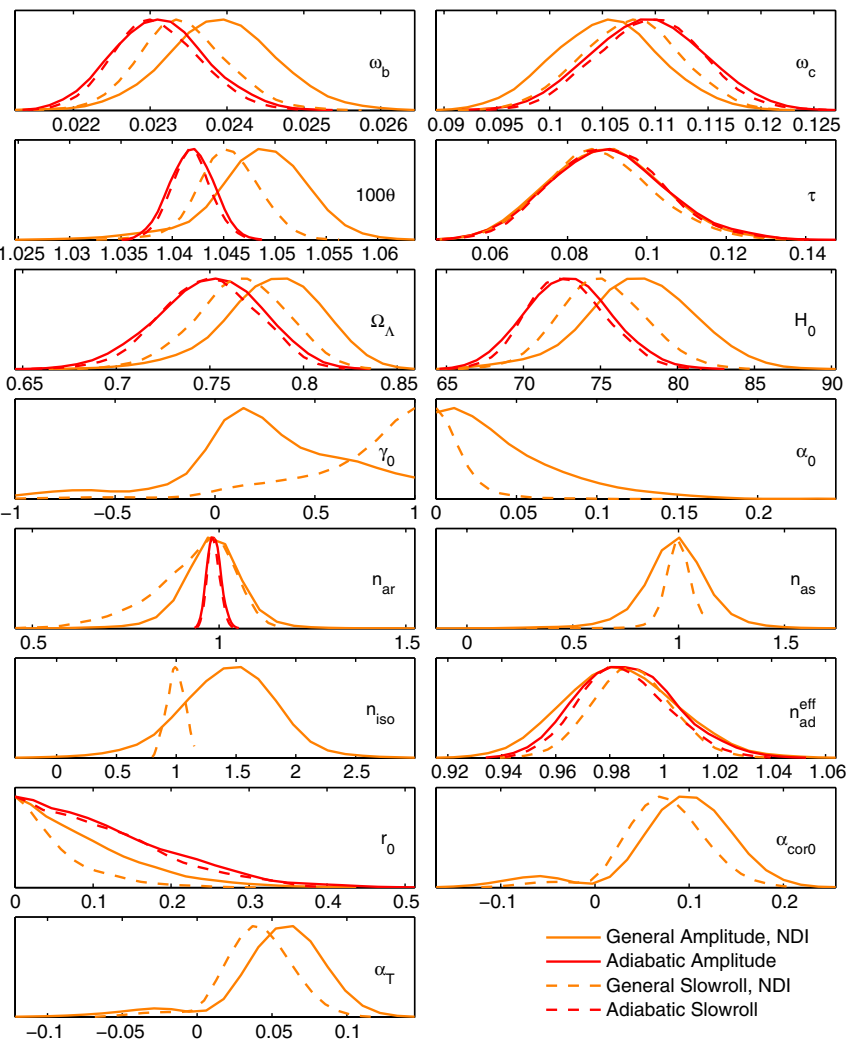

FIG. 7 (color online). Comparison of amplitude and slow-roll parametrizations, general correlation. Marginalized 1d posterior pdfs from MULTINEST runs made in the phenomenological amplitude parametrization (solid lines) and inflationary slow-roll parametrization (dashed lines) with a generally correlated mixture of primordial adiabatic and NDI modes (orange/gray) and with the pure adiabatic mode (red/black).

since the positive correlation adds power at low $\ell$. Thus there is less room for the tensor contribution, which would also add power at low $\ell$.

Of the four slow-roll parameters only $\varepsilon$ is well constrained in all studied cases, while the three $\eta_{i j}$ are unconstrained or very poorly constrained, except $\eta_{\sigma \sigma}$ in the adiabatic case. The constraint on $\varepsilon$ does not come only from the tensor contribution. From the first line of Eq. (24) it is obvious that if $\varepsilon$ was near the upper bound (0.075) of our chosen prior range, this would lead to a too red-tilted adiabatic spectrum, which cannot be compensated by the blue-tilted isocurvature spectrum at high $\ell$ since the third line of Eq. (24) gives $0.70<$ $n_{\text {iso }}<1.15$.

In the slow-roll parametrization the adiabatic model has $-\ln Z \approx 3,898.9$, whereas the mixed models (with general correlation) have $-\ln Z \approx 3,901.5$ (NDI) and $-\ln Z \approx 3,902.9$ (CDI). So the betting odds in favor of the adiabatic model are 13:1 against NDI, and 51:1 against CDI. In particular, the mixed NDI case is not overwhelmingly disfavored by the Bayesian model comparison when the slow-roll approach is adopted.

\section{SPECIAL CASES}

Now we study uncorrelated $(\gamma=0)$ and maximally correlated $(\gamma=1)$ or anticorrelated $(\gamma=-1)$ cases. The uncorrelated model has only two extra parameters compared to the "standard" adiabatic $\Lambda \mathrm{CDM}$ model. In the maximally correlated cases, in the amplitude parametrization, we make an extra assumption that the adiabatic and isocurvature spectra have the same shape, which further reduces the number of parameters by 1 , leading to only one extra parameter. In the slow-roll parametrization the same shape of spectra follows directly from the second and third lines of Eq. (24).

\section{A. Uncorrelated case for NDI, NVI, and CDI}

In the uncorrelated case the $\mathcal{P}_{\text {as }}(k)$ spectrum is absent, so we have only nine independent parameters: the four background parameters

$$
\omega_{b}, \omega_{c}, \theta, \tau,
$$

and five perturbation parameters

$$
\ln A_{0}^{2}, n_{\mathrm{ar}}, \alpha_{0}, n_{\mathrm{iso}}, r_{0},
$$

where

$$
\begin{gathered}
n_{\mathrm{ar}}=1-6 \varepsilon+2 \eta_{\sigma \sigma}, \quad n_{\text {iso }}=1-2 \varepsilon+2 \eta_{s s}, \\
r_{0}=16 \varepsilon .
\end{gathered}
$$

The primary perturbation parameters in the amplitude parametrization are

$$
\ln A_{1}^{2}, \ln A_{2}^{2}, \alpha_{1}, \alpha_{2}, \tilde{r}_{0}=r_{0},
$$

and in the slow-roll parametrization

$$
\ln A_{0}^{2}, \alpha_{0}, \eta_{\sigma \sigma}, \eta_{s s}, \varepsilon
$$

The marginalized 1d posterior pdfs are indicated in Fig. 8 by $\gamma=0$ curves for NDI, in Fig. 9 by $\gamma=0$ curves for NVI in the amplitude parametrization, and in Figs. 10 and 11 by $\gamma=0$ curves for NDI in the slow-roll parametrization. (Again, we drop the NVI case from the slow-roll analysis as it is hard to motivate.) To allow for an easy comparison, we also plot the generally correlated and adiabatic cases presented in the previous section. Numerical results for the $\gamma=0$ case are reported in the fourth columns of Tables II, III, IV, V, and VI.

On all scales in all cases the allowed primordial isocurvature fraction $\left(\alpha_{1,2,0}\right)$ is much larger in the uncorrelated case than in the generally correlated case or in the maximally correlated cases $\gamma= \pm 1$ (studied in the next subsection). In the other models the main nonadiabatic effect comes from the correlation (whose amplitude is somewhere between the adiabatic and isocurvature contributions), but in the uncorrelated case the only disturbance to the adiabatic spectrum comes from the isocurvature itself. Thus rather large primordial fractions can be accommodated by the 


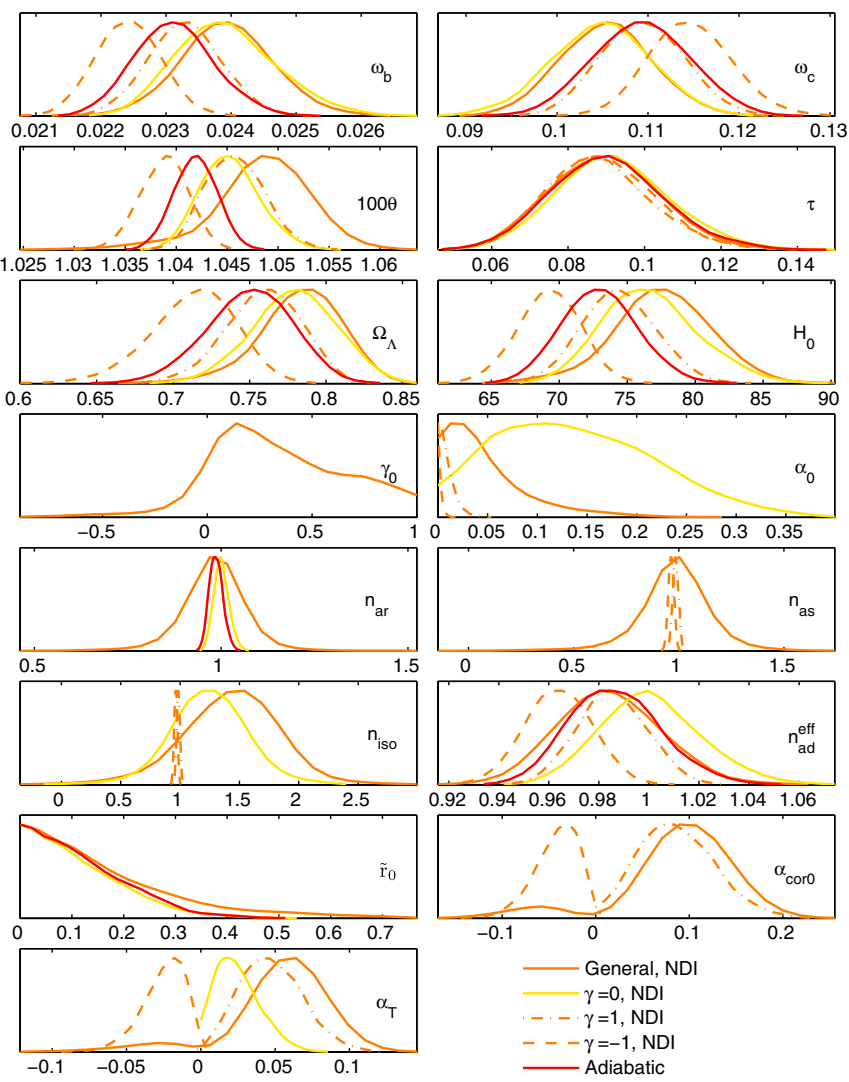

FIG. 8 (color online). Amplitude parametrization, comparison of special and general NDI cases. Marginalized 1d pdfs for a generally correlated mixture of primordial adiabatic and NDI (solid orange/gray) modes compared to the uncorrelated ( $\gamma=0$, solid yellow/light gray), maximally correlated $(\gamma=1$, dotdashed orange/gray), and maximally anticorrelated $(\gamma=-1$, dashed orange/gray) cases, as well as to the pure adiabatic model (solid red/black).

CMB data. However, since the isocurvature component is more off-phase from the adiabatic one than the correlation component, the allowed nonadiabatic contribution $\left|\alpha_{T}\right|$ to the observed $\mathrm{CMB}$ temperature variance is smaller in all uncorrelated cases than in the general cases.

In the uncorrelated NDI and CDI cases the slow-roll parameters $\eta_{\sigma \sigma}$ and $\epsilon$ are constrained equally well as in the adiabatic case, whereas $\eta_{s s}$ remains unconstrained. A small isocurvature contribution at low $\ell$ is allowed by the data (in particular due to cosmic variance), and the value of $n_{\text {iso }}$ does not matter as long as the spectrum is nearly scale invariant (as it is due to our chosen priors of the slow-roll parameters). Since $r_{0}$ is simply 16 times $\varepsilon$, we find a tight constraint on the tensor-to-scalar ratio. Indeed, we find $r_{0}<0.25$ in both the uncorrelated NDI and CDI cases, while the pure adiabatic case leads to $r_{0}<0.31$ at 95\% C.L. The tightening of the constraint on $r_{0}$ when allowing for the uncorrelated isocurvature component is natural, since adding power at low $\ell$ reduces room from the tensor contribution.

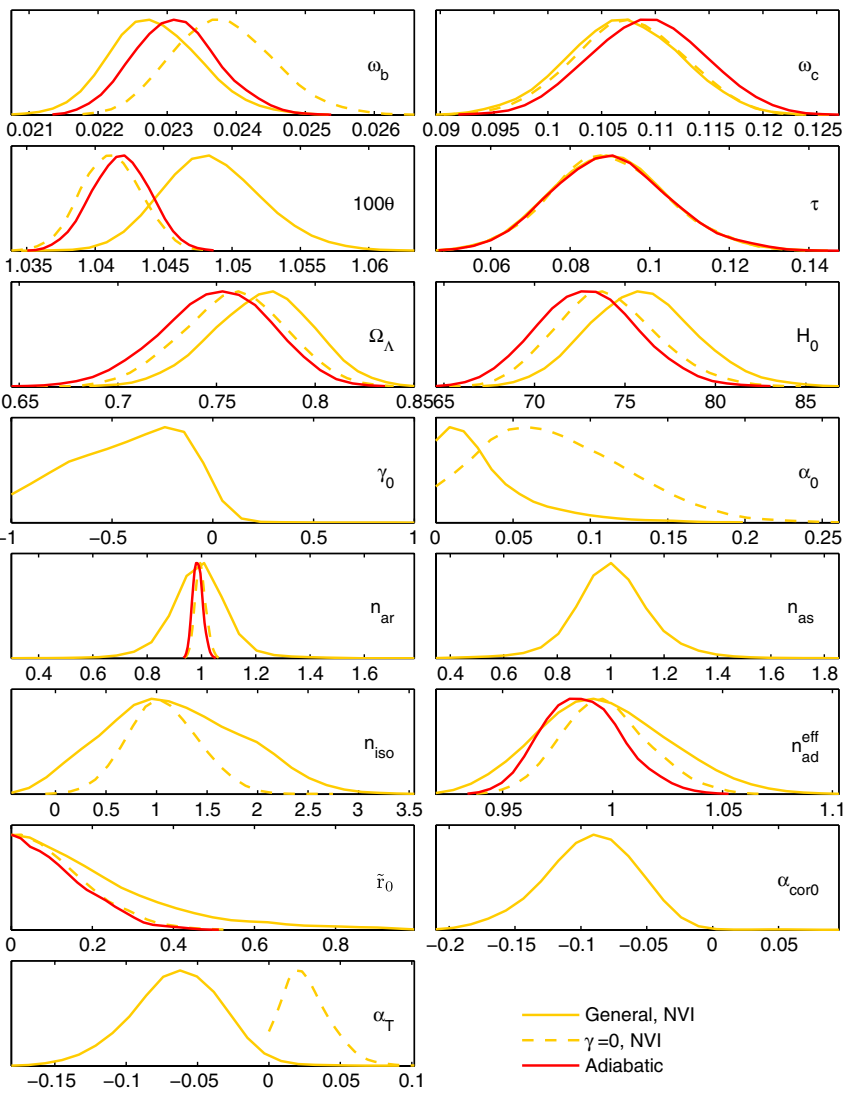

FIG. 9 (color online). Amplitude parametrization, comparison of special and general NVI cases. Marginalized 1d pdfs for a generally correlated mixture of primordial adiabatic and NVI (solid yellow/light gray) modes compared to the uncorrelated ( $\gamma=0$, dashed yellow/light gray) case and to the pure adiabatic model (solid red/black).

The betting odds in favor of the adiabatic model are 3.4, 16, 5.2, 3.5, and 16:1 when compared to the NDI (ampl. par.), NVI (ampl. par.), CDI (ampl. par.), NDI (slow-roll), CDI (slow-roll) models, respectively. Indeed, out of all models studied in this paper, the uncorrelated mixed NDI model in amplitude parametrization turns out to be least disfavored compared to the adiabatic model in terms of Bayesian model comparison.

\section{B. Maximally (anti)correlated NDI or CDI}

In the fully correlated cases the $\mathcal{P}_{\text {ar }}(k)$ spectrum is absent, and, following [1], we assume there are no tensor perturbations. Moreover, according to Eq. (24), the twofield slow-roll inflation gives

$$
n_{\mathrm{as}}=n_{\mathrm{iso}}=1+2\left(\eta_{s s}-\varepsilon\right) .
$$

No matter what $\varepsilon$ is, these models lead to zero tensor contribution. Thus we can only constrain the combination $\eta_{s s}-\varepsilon$, not $\eta_{s s}$ and $\varepsilon$ individually. Unlike in [1], we assume $n_{\text {as }}=n_{\text {iso }}$ also in the amplitude parametrization in order to make comparison to slow-roll results more 


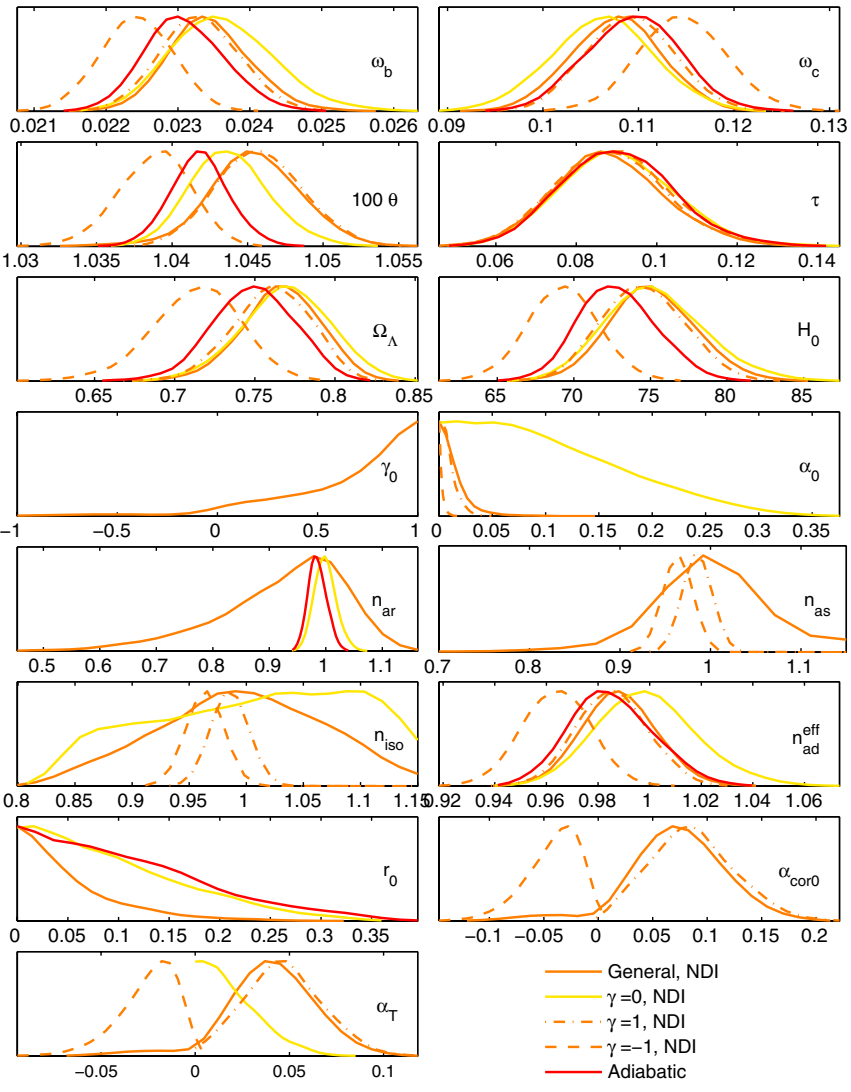

FIG. 10 (color online). Slow-roll parametrization, comparison of special and general NDI cases. Marginalized 1d pdfs for a generally correlated mixture of primordial adiabatic and NDI (solid orange/gray) modes compared to the uncorrelated ( $\gamma=0$, solid yellow/light gray), maximally correlated $(\gamma=1$, dotdashed orange/gray), and maximally anticorrelated $(\gamma=-1$, dashed orange/gray) cases, as well as to the pure adiabatic model (solid red/black).

straightforward and to have the same number of parameters in both parametrizations, which affects the Bayesian model comparison results.

Therefore, we have only seven independent parameters: background parameters

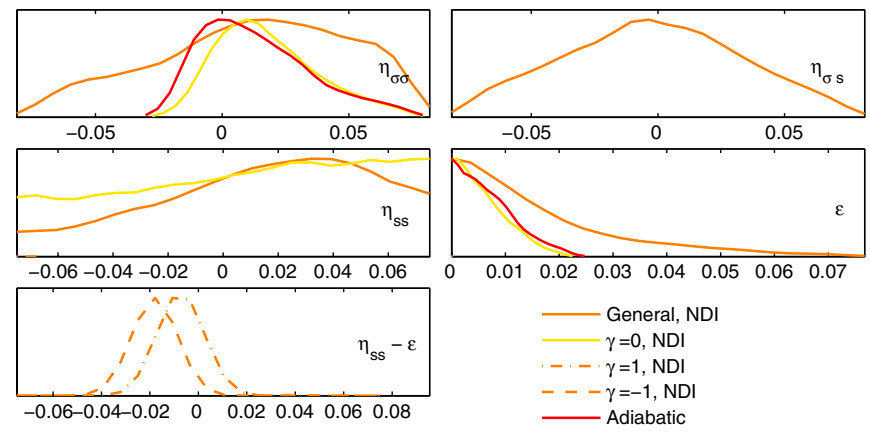

FIG. 11 (color online). Slow-roll parametrization, comparison of slow-roll parameters in special and general NDI cases. Marginalized 1d posterior probability densities of the primary slow-roll parameters. The line styles are the same as in Fig. 10. $\omega_{b}, \omega_{c}, \theta, \tau$,

and three perturbation parameters

$$
\ln A_{0}^{2}, n_{\text {iso }}=n_{\mathrm{as}}, \alpha_{0} .
$$

Since $n_{\text {as }}=n_{\text {iso }}$ (and the "ar" component is missing), the primordial isocurvature fraction is scale independent, $\alpha=\alpha_{1,2}=\alpha_{0}$.

The primary perturbation parameters in the amplitude parametrization are

$$
\ln A_{1}^{2}, \ln A_{2}^{2}, \alpha_{1}\left(=\alpha_{2}\right),
$$

and in the slow-roll parametrization

$$
\ln A_{0}^{2}, \alpha_{0}, \eta_{s s}-\varepsilon .
$$

The marginalized $1 \mathrm{~d}$ posterior pdfs of the NDI case are indicated by the dashed magenta $(\gamma=-1,100 \%$ anticorrelation) and dot-dashed magenta $(\gamma=+1,100 \%$ correlation) curves in Fig. 8 for the amplitude parametrization, and in Figs. 10 and 11 for the slow-roll parametrization. The numerical values are tabulated in the fifth and sixth columns of Tables II (NDI) and IV (CDI) in the amplitude parametrization, and in Tables V (NDI) and VI (CDI) in the slow-roll parametrization. Note that we do not study the mixed NVI model with maximal (anti) correlation, since it is hard to think of any physical mechanism that would lead to a correlation between NVI and adiabatic perturbations since they may originate from very different epochs of the evolution of the universe.

In the amplitude parametrization, we obtain very tightly constrained isocurvature fraction $\alpha$ for both the NDI and CDI cases, $\gamma= \pm 1$. The $95 \%$ C.L. limits for NDI are $\alpha<0.0303$ and $\alpha<0.0093$ for $\gamma=+1$ and $\gamma=-1$, respectively. Since the data force the adiabatic spectrum to be nearly scale invariant, and $n_{\text {iso }}=n_{\text {as }}$, we would expect very little difference from the slow-roll case, and indeed we find in the slow-roll parametrization very similar results, $\alpha<0.0280$ and $\alpha<0.0104$ for $\gamma=+1$ and $\gamma=-1$, respectively. The reason for these tight constraints is that in the maximally correlated cases a fixed value of $\alpha$ leads to much larger nonadiabatic contributions than in the partially correlated cases. As we would expect, in the cases where the maximal correlation has the same sign that was preferred in the general case, the constraints on $\alpha_{\text {cor0 } 0}$ and $\alpha_{T}$ are very similar between the maximally correlated and generally correlated models.

The only "slow-roll parameter" of the maximally correlated cases, the combination $\eta_{s s}-\varepsilon$, is well constrained.

The Bayesian evidences for the maximally correlated models are in all cases worse than for the uncorrelated models where we found the best evidences compared to the adiabatic model. 


\section{COMPARISON TO PLANCK RESULTS}

In the literature, the CDI modes have been extensively contrasted against observations, but the observational constraints on neutrino isocurvature have been studied less, although theoretical work and future forecasts can be found in many publications; see, e.g., [11,90-93]. In this section we compare our results to the most recent constraints on the phenomenological mixed CDI, NDI, and NVI models that come from the Planck temperature anisotropy data; see [6].

We summarize the Planck and our WMAP-9, ACBAR and QUaD ("WMAP") constraints on the key isocurvature variables in Table I. Perhaps surprisingly, the Planck constraints on the primordial isocurvature fraction in generally correlated mixed models are weaker than what we find here, in particular at large scales. For example, the Planck constraint $\alpha_{1}<0.27$ in the NDI model is almost a factor of 3 weaker than our constraint $\alpha_{1}<0.10$.

The reason for such an unexpected difference between WMAP-9 and Planck is that the Planck data seem to prefer a negative correlation due to a relatively low power at low multipoles $\ell \sim 2 \ldots 40$ compared to the higher multipoles. The adiabatic $\Lambda \mathrm{CDM}$ model fits the acoustic peak structure of the Planck data with high precision, but even the best-fitting adiabatic model leads to more power at low $\ell$ than seen in the data. This leads to a "demand" of some power-reducing mechanism at low $\ell$; a negatively correlated isocurvature mode can provide such an effect. This explains why the Planck constraints are weaker than the WMAP-9 constraints at large scales and why Planck prefers negative correlation for all three cases (CDI, NDI, NVI), whereas WMAP-9 prefers a positive correlation in the CDI and NDI cases and a negative correlation in the NVI case (since with WMAP-9 the main nonadiabatic effects come from the first acoustic peak region).

Another crucial difference between WMAP-9 and Planck is that the Planck data prefer smaller $H_{0}$ and $\Omega_{\Lambda}$ and constrain the background parameters much more tightly, thus leaving less freedom to play with their values. The WMAP-9 data prefer very large $H_{0}$ and $\Omega_{\Lambda}$ in the phenomenological mixed models in order to compensate the shift of the first acoustic peak to the right caused by the nonadiabatic component.

Some of the differences between Planck and our WMAP-9 results may come from the different parametrizations and assumptions. In [6] the curvature (i.e., adiabatic) spectrum was described by a power law, the isocurvature by an other one, and the correlation by a third one power law, which had a kink either at the low- $k$ or at the high- $k$ region to keep $\left|\mathcal{C}_{\mathcal{R} S}(k)\right| \leq \sqrt{\mathcal{P}_{\mathcal{R}}(k) \mathcal{P}_{S}(k)}$. Instead, we assume two powerlaw components for the curvature perturbation, of which the other is fully correlated with the isocurvature power spectrum. This leads our curvature spectrum to "auto-adjust" (run) in such a way that the above-mentioned mathematically necessary condition is always automatically satisfied without introducing kinks to any of the spectra. Moreover, we use the relative amplitudes $\alpha_{1,2}$ and $\gamma_{1,2}$ and the logarithm of the "total" amplitudes $\ln \left(10^{10} A_{1,2}^{2}\right)$ as primary parameters, but in [6] the actual amplitudes of the three power spectra at two scales were primary parameters. We also include tensor perturbations in the analysis, while in [6] the isocurvature analysis was done without tensor perturbations. However, based on [1] we do not expect this to cause major differences.

In the last two rows of Table I we compare the constraints in two special cases: the maximally correlated and anticorrelated CDI. (The uncorrelated cases are not comparable, since we allow for a free $n_{\text {iso }}$, whereas in [6] $n_{\text {iso }}$ is fixed to unity.) The maximally (anti)correlated cases are the most directly comparable, since we do not allow for a tensor contribution in this case and both [6] and this paper assume that the adiabatic and isocurvature spectral indices are equal. After the above discussion of the generally correlated cases, it is not a surprise that the Planck data lead to extremely tight constraints in the maximally correlated case, and constraints weaker than the WMAP in the maximally anticorrelated case. This reinforces the conclusion that the Planck data disfavor any contribution that would increase the temperature angular power at large scales (low $\ell$ ) over the one obtained in the adiabatic $\Lambda \mathrm{CDM}$ model, and indeed favor a negative contribution.

TABLE I. Comparison of Planck [6] and WMAP (this paper) 95\% C.L. isocurvature constraints in phenomenological parametrizations. The first three rows are for the generally correlated mixed models and the last two rows for the maximally correlated $(\gamma=1)$ or anticorrelated $(\gamma=-1)$ CDI.

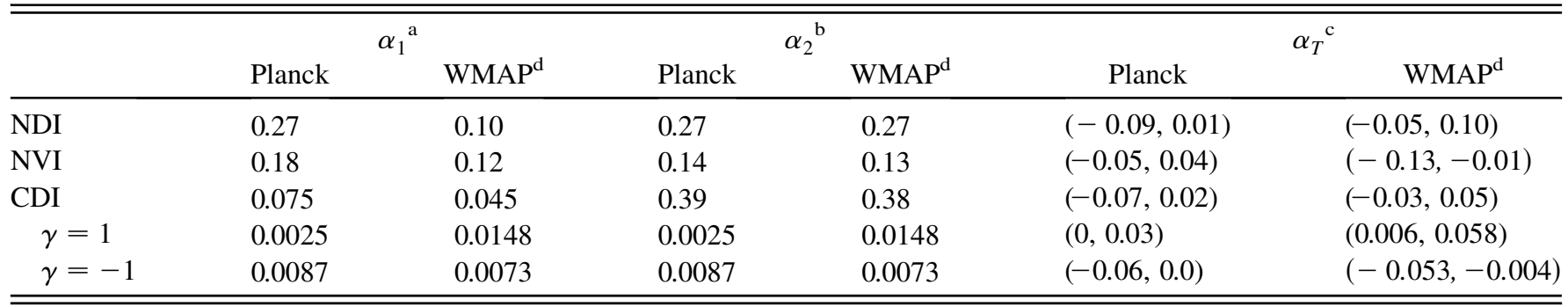

The primordial isocurvature fraction at $k=0.002 \mathrm{Mpc}^{-1}$ is called $\beta_{\text {iso }}\left(k_{\text {low }}\right)$ in [6].

${ }^{\mathrm{b}}$ The primordial isocurvature fraction at $k=0.050 \mathrm{Mpc}^{-1}$ is called $\beta_{\text {iso }}\left(k_{\text {mid }}\right)$ in [6].

${ }^{\mathrm{c}}$ The nonadiabatic contribution to the CMB temperature variance, $\alpha_{T}$, can be calculated from [6] as $1-\alpha_{\mathcal{R} R}^{(2,2500)}$.

dOur results with WMAP-9 [2], ACBAR [4] and QUaD [5] data. 


\section{DISCUSSION}

We constrained the primordial fraction of all regular isocurvature modes, one at a time (matter density, neutrino density, and neutrino velocity isocurvature), as well as the nonadiabatic contribution to the observed CMB temperature variance. As the matter density (or CDI) mode has been extensively studied previously, our focus was on the neutrino isocurvature modes (NDI, NVI), but we also updated the constraints on CDI. Since primordial tensor perturbations are produced in typical inflationary models, we included tensor perturbations throughout the analysis.

In the phenomenological approach the power-law spectra of the curvature and isocurvature modes, and the correlation between them, had independent amplitudes and tilts. This added four independent perturbation parameters to the standard adiabatic flat $\Lambda \mathrm{CDM}$ scenario. If any clear nonadiabatic features were present in the CMB data used (WMAP-9, ACBAR, and QUaD), this approach should have found them. Neither frequentist nor Bayesian methods indicated any preference for any of the isocurvature modes: the CMB data set tight upper bounds on nonadiabatic contributions to the observed temperature variance.

Using Bayesian evidences calculated by MULTINEST we established the betting odds for the models studied. For the generally correlated mixture of the adiabatic and one isocurvature mode (either NDI, NVI, or CDI) compared to the pure adiabatic primordial perturbation mode, we found the odds to be as small as 1:100. However, in the special cases where we imposed restrictions on the correlation component, the betting odds were higher. In particular, for an uncorrelated mixed NDI model (which had two nonadiabatic extra parameters) the betting odds were 1:3.4 compared to the pure adiabatic model.

In the phenomenological setup, with generally correlated mixed adiabatic and isocurvature perturbations, the tensor perturbations have been included at least in two different previous publications, in [21] for NDI, NVI, and CDI, and in [1] for CDI. In both of these, the first inflationary consistency relation was used in order to reduce the number of extra parameters. Namely, the tensor spectral index was determined from the consistency relation, $n_{T}=-r /[8(1-|\gamma|)]$, where $r$ was the tensor-to-scalar ratio (the ratio of tensor and total curvature perturbation power at the primordial time) and $\gamma$ the correlation fraction. In both [1,21], assigning uniform priors on $r$ and $\gamma$ (or $\sqrt{1-|\gamma|}$ ) led to "tight" constraints on $\gamma$. This was due to an unphysical prior of $n_{T}$ : whenever $|\gamma|$ was near 1 , the tensor spectral index was very negative. The huge tensor contribution thus induced was disfavored by the low- $\ell$ data. However, the original idea behind using the consistency relation was to obtain physically motivated (near zero) values for $n_{T}$ as predicted by generic inflationary models, not huge negative values. In this paper we, for the first time, addressed these problems. We introduced the tensor-to-scalar ratio at horizon exit during inflation, $\tilde{r}$. This is related to the above definition by $r=\tilde{r}(1-|\gamma|)$. The inflationary consistency relation (derived to first order in slow-roll parameters) now reads $n_{T}=$ $-\tilde{r} / 8$, and this led to a uniform prior on $n_{T}$ between $\min (\tilde{r})$ and zero, avoiding unphysical values and, most importantly, avoiding the interference of the use of the consistency relation with the constraints on $\gamma$. The difference between the "old" and new approaches was presented in Fig. 4.

We studied the matter and neutrino density modes also in the two-field slow-roll inflation context, where we assumed uniform priors on the four first order slow-roll parameters $\left(\varepsilon, \eta_{\sigma \sigma}, \eta_{\sigma s}, \eta_{s s}\right)$ and assumed their magnitude to be small, i.e., less than 0.075 , so that the slow-roll approximation was accurate enough. The main difference from the phenomenological approach came from the fact that the choice of prior ranges of the slow-roll parameters restricted all the primordial spectra to be nearly scale invariant. (In the phenomenological approach the data favored blue-tilted isocurvature spectra with spectral indices $n_{\text {iso }} \sim 1.45$ for NDI or $n_{\text {iso }} \sim 2.05$ for CDI, which in the slow-roll approach were excluded by the prior.)

In all those slow-roll cases, where tensor perturbations were produced, the posterior probability density of $\varepsilon$ was much narrower than its prior. In the models with generally correlated primordial adiabatic and CDI or NDI modes, the constraint on $\varepsilon$ was weaker than in the pure adiabatic model, but in the models with uncorrelated adiabatic and CDI or NDI modes the constraint on $\varepsilon$ was tighter since the only possible effect (on the temperature angular power) of the nearly scale-invariant uncorrelated isocurvature component was to add power to the low multipoles, where the tensor contribution would also add power. Unlike $\varepsilon$, all three $\eta_{i j}$ parameters were weakly constrained or unconstrained: since our slow-roll approach led to almost scale-invariant isocurvature and correlation spectra, the "nonadiabatic" modifications to $C_{\ell}$ appeared only in the low- $\ell$ region, which is cosmic variance dominated and hence insensitive to the small tilts of the isocurvature and correlation components.

In Sec. III, assuming a curvaton-type model with inhomogeneous lepton asymmetry, and taking into account the big bang nucleosynthesis constraint on the neutrino asymmetry and the Planck constraint on non-Gaussianity (and converting this to a constraint of the curvaton inertia fraction $R$ at its decay time), we derived an upper limit for the primordial isocurvature fraction, $\alpha<0.0045$, within this specific maximally correlated neutrino density isocurvature model. The direct constraint from the CMB, $\alpha<0.0256$ (see Sec. VIB for the constraints on the NDI $\gamma=1$ case in the amplitude parametrization), is weaker by a factor 6 .

In [1] we studied the CDI mode in a similar setup as NDI, NVI, and CDI here. We have checked the consistency of our new CDI results against the old ones. The differences can be traced to the following three points: different parametrization for the tensor-to-scalar ratio (in the phenomenological approach), different data (i.e., WMAP-9 versus WMAP-7), and an updated recombination code RECFAST in CAMB. 
In Sec. VII we compared the Planck [6] isocurvature analysis to our WMAP-based analysis. We discussed the unexpected result that WMAP seems to constrain isocurvature more tightly than Planck. This is due to the lack of power at low multipoles, $\ell \sim 2 \ldots 40$, in the Planck data compared to the prediction of the best-fitting adiabatic $\Lambda \mathrm{CDM}$ model. Hence the Planck data prefer a power-reducing mechanism, which the mixed adiabatic and isocurvature models with negative correlation or full anticorrelation can offer. In the near future, it will be interesting to see whether the Planck low- $\ell$ polarization data support the negatively correlated isocurvature contribution or agree better with the pure adiabatic model. (It should be kept in mind that the current Planck isocurvature analysis in [6] used the WMAP polarization data.)

Moreover, the (Planck) high- $\ell$ polarization data will be valuable, since the different phases of isocurvature and adiabatic modes are not only imprinted in the temperature angular power but also in the polarization $E$-mode. In the longer term, as discussed in [1], the measurement of baryon acoustic oscillations at the precision forecasted for the Euclid mission [94] may provide further constraints or a detection (for more details, see, e.g., $[92,95]$ ), in particular, if the isocurvature spectral index $n_{\text {iso }}$ is larger than the adiabatic spectral index. Apart from these direct constraints set by the amplitudes and phases of perturbations, any measurements that tighten or shift the constraints on the background parameters, such as $\omega_{c}, H_{0}, \Omega_{\Lambda}$, will affect the constraints on isocurvature modes.

\section{ACKNOWLEDGMENTS}

This work was supported by the Academy of Finland Grant No. 257989. The authors were granted access to the HPC resources of CSC made available within the Distributed European Computing Initiative by the PRACE-2IP, receiving funding from the European Community's Seventh Framework Programme (FP7/2007-2013) under Grant Agreement No. RI-283493. We thank the CSC-Scientific Computing Ltd. (Finland) for computational resources. M. S. was supported by the Magnus Ehrnrooth Foundation, and S. R. by the Väisälä Foundation.

\section{APPENDIX: TABLES}

In this section we tabulate numerical constraints on cosmological parameters obtained in the amplitude parametrization for the NDI, NVI, and CDI models and in the slow-roll parametrization for NDI and CDI models. In each table we show the adiabatic model for comparison.

TABLE II. Amplitude parametrization, NDI. The median values and $68 \%$ or $95 \%$ C.L. intervals (in parentheses) are given for a selection of parameters. For the fully (anti)correlated models, $\gamma= \pm 1$, we have $r_{0}=0$, so these models are without a tensor contribution. The last line, $Z_{\text {adiab }} / Z$, shows the ratio of the probability of the pure adiabatic model compared to the model of each column.

\begin{tabular}{|c|c|c|c|c|c|c|}
\hline Parameter & C.L & Mixed NDI, general & Mixed NDI, $\gamma=0$ & Mixed NDI, $\gamma=1$ & Mixed NDI, $\gamma=-1$ & Adiabatic \\
\hline$\omega_{b}$ & $68 \%$ & $0.0239(0.0233,0.0246)$ & $0.0239(0.0232,0.0247)$ & $0.0233(0.0227,0.0238)$ & $0.0224(0.0219,0.0229)$ & $0.0231(0.0225,0.0237)$ \\
\hline$\omega_{c}$ & $68 \%$ & $0.1054(0.1004,0.1104)$ & $0.1049(0.0995,0.1102)$ & $0.1094(0.1048,0.1140)$ & $0.1145(0.1102,0.1191)$ & $0.1093(0.1040,0.1146)$ \\
\hline $100 \theta$ & $68 \%$ & $1.0488(1.0447,1.0526)$ & $1.0452(1.0425,1.0482)$ & $1.0457(1.0428,1.0486)$ & $1.0389(1.0364,1.0411)$ & $1.0419(1.0398,1.0440)$ \\
\hline$\tau$ & $68 \%$ & $0.0896(0.0753,0.1035)$ & $0.0911(0.0776,0.1065)$ & $0.0872(0.0746,0.1011)$ & $0.0884(0.0756,0.1023)$ & $0.0899(0.0757,0.1045)$ \\
\hline$\Omega_{\Lambda}$ & $68 \%$ & $0.7852(0.7586,0.8090)$ & $0.7800(0.7525,0.8070)$ & $0.7602(0.7346,0.7835)$ & $0.7147(0.6865,0.7388)$ & $0.7508(0.7221,0.7769)$ \\
\hline$H_{0}$ & $68 \%$ & $77.56(74.31,81.01)$ & $76.49(73.35,80.16)$ & $74.35(71.69,77.11)$ & $69.28(67.02,71.47)$ & $72.95(70.27,75.69)$ \\
\hline $\ln \left[10^{10} A_{0}^{2}\right]$ & $68 \%$ & $3.0713(3.0260,3.1323)$ & $3.2032(3.1360,3.2840)$ & $3.0717(3.0342,3.1120)$ & $3.1649(3.1300,3.2067)$ & $3.1038(3.0641,3.1422)$ \\
\hline$n_{\text {ad }}^{\text {eff }}$ & $68 \%$ & $0.9835(0.9640,1.0042)$ & $0.9997(0.9811,1.0204)$ & $0.9850(0.9718,0.9992)$ & $0.9638(0.9509,0.9769)$ & $0.9859(0.9685,1.0041)$ \\
\hline$\gamma_{1}$ & $95 \%$ & $>-0.1137$ & & & & \\
\hline$\gamma_{2}$ & $95 \%$ & $>-0.0894$ & & & & \\
\hline$\alpha_{1}$ & $95 \%$ & $<0.0980$ & $<0.2421$ & $<0.0303$ & $<0.0093$ & \\
\hline$\alpha_{2}$ & $95 \%$ & $<0.2713$ & $<0.4003$ & $<0.0303$ & $<0.0093$ & \\
\hline$\tilde{r}_{0}$ & $95 \%$ & $<0.4751$ & $<0.2866$ & & & $<0.3334$ \\
\hline$\gamma_{0}$ & $95 \%$ & $>-0.1004$ & & & & \\
\hline$\alpha_{0}$ & $95 \%$ & $<0.1414$ & $<0.2816$ & $<0.0303$ & $<0.0093$ & \\
\hline$n_{\mathrm{ar}}$ & $95 \%$ & $0.9846(0.8025,1.1361)$ & $0.9997(0.9651,1.0427)$ & & & $0.9859(0.9545,1.0236)$ \\
\hline$n_{\text {as }}$ & $95 \%$ & $0.9837(0.6240,1.2896)$ & & $0.9850(0.9593,1.0116)$ & $0.9638(0.9377,0.9889)$ & \\
\hline$n_{\text {iso }}$ & $95 \%$ & $1.4508(0.5678,2.1718)$ & $1.2465(0.6096,1.8885)$ & $0.9850(0.9593,1.0116)$ & $0.9638(0.9377,0.9889)$ & \\
\hline$r_{0}$ & $95 \%$ & $<0.2414$ & $<0.2866$ & & & $<0.3334$ \\
\hline$\alpha_{\text {cor } 0}$ & $95 \%$ & $0.0961(-0.0824,0.1808)$ & & $0.0846(0.0184,0.1713)$ & $-0.0389(-0.0960,-0.0067)$ & \\
\hline & $95 \%$ & $0.0580(-0.0486,0.1028)$ & $<0.0531$ & $0.0462(0.0104,0.0916)$ & $-0.0230(-0.0593,-0.0039)$ & \\
\hline$-\ln Z$ & & 3905.78 & 3902.38 & 3902.73 & 3905.35 & 3901.17 \\
\hline $\ln \left(Z_{\text {adiab }} / Z\right)$ & & 4.6 & 1.2 & 1.6 & 4.2 & 0 \\
\hline$Z_{\text {adiab }} / Z$ & & 100 & 3.4 & 4.8 & 65 & 1 \\
\hline
\end{tabular}


TABLE III. Amplitude parametrization, NVI. The median values and $68 \%$ or $95 \%$ C.L. intervals (in parentheses) are given for a selection of parameters.

\begin{tabular}{|c|c|c|c|c|}
\hline Parameter & C.L & Mixed NVI, general corr. & NVI, $\gamma=0$ & Adiabatic \\
\hline$\omega_{b}$ & $68 \%$ & $0.0228(0.0222,0.0235)$ & $0.0238(0.0231,0.0245)$ & $0.0231(0.0225,0.0237)$ \\
\hline$\omega_{c}$ & $68 \%$ & $0.1071(0.1020,0.1122)$ & $0.1075(0.1024,0.1126)$ & $0.1093(0.1040,0.1146)$ \\
\hline $100 \theta$ & $68 \%$ & $1.0484(1.0451,1.0521)$ & $1.0412(1.0390,1.0433)$ & $1.0419(1.0398,1.0440)$ \\
\hline$\tau$ & $68 \%$ & $0.0898(0.0760,0.1040)$ & $0.0893(0.0765,0.1041)$ & $0.0899(0.0757,0.1045)$ \\
\hline$\Omega_{\Lambda}$ & $68 \%$ & $0.7744(0.7482,0.7984)$ & $0.7596(0.7326,0.7840)$ & $0.7508(0.7221,0.7769)$ \\
\hline$H_{0}$ & $68 \%$ & $75.89(73.05,78.85)$ & $73.87(71.33,76.70)$ & $72.95(70.27,75.69)$ \\
\hline $\ln \left[10^{10} A_{0}^{2}\right]$ & $68 \%$ & $3.1895(3.1399,3.2402)$ & $3.1462(3.1005,3.1930)$ & $3.1038(3.0641,3.1422)$ \\
\hline$n_{\text {ad }}^{\text {eff }}$ & $68 \%$ & $0.9948(0.9680,1.0265)$ & $0.9958(0.9777,1.0171)$ & $0.9859(0.9685,1.0041)$ \\
\hline$\gamma_{1}$ & $95 \%$ & $<-0.0588$ & & \\
\hline$\gamma_{2}$ & $95 \%$ & $<-0.0604$ & & \\
\hline$\alpha_{1}$ & $95 \%$ & $<0.1245$ & $<0.1961$ & \\
\hline$\alpha_{2}$ & $95 \%$ & $<0.1349$ & $<0.1976$ & \\
\hline$\tilde{r}$ & $95 \%$ & $<0.6213$ & $<0.3088$ & $<0.3334$ \\
\hline$\gamma_{0}$ & $95 \%$ & $<-0.0637$ & & \\
\hline$\alpha_{0}$ & $95 \%$ & $<0.0997$ & $<0.1617$ & \\
\hline$n_{\mathrm{ar}}$ & $95 \%$ & $0.9935(0.7785,1.1985)$ & $0.9958(0.9607,1.0390)$ & $0.9859(0.9545,1.0236)$ \\
\hline$n_{\mathrm{as}}$ & $95 \%$ & $1.0009(0.7213,1.3083)$ & & \\
\hline$n_{\text {iso }}$ & $95 \%$ & $1.1454(0.0329,2.4865)$ & $1.0528(0.4302,1.7779)$ & \\
\hline$r_{0}$ & $95 \%$ & $<0.2489$ & $<0.3088$ & $<0.3334$ \\
\hline$\alpha_{\text {cor0 } 0}$ & $95 \%$ & $-0.0907(-0.1636,-0.0333)$ & & \\
\hline$\alpha_{T}$ & $95 \%$ & $-0.0635(-0.1267,-0.0091)$ & $<0.0576$ & \\
\hline$-\ln Z$ & & 3905.57 & 3903.96 & 3901.17 \\
\hline $\ln \left(Z_{\text {adiab }} / Z\right)$ & & 4.4 & 2.8 & 0 \\
\hline$Z_{\text {adiab }} / Z$ & & 81 & 16 & 1 \\
\hline
\end{tabular}

TABLE IV. Amplitude parametrization, CDI. The median values and 68\% or 95\% C.L. intervals (in parentheses) are given for a selection of parameters. For the fully (anti)correlated models, $\gamma= \pm 1$, we have $r_{0}=0$, so these models are without a tensor contribution.

\begin{tabular}{|c|c|c|c|c|c|c|}
\hline Parameter & C.L & Mixed CDI, general corr. & Mixed CDI, $\gamma=0$ & Mixed CDI, $\gamma=1$ & Mixed CDI, $\gamma=-1$ & Adiabatic \\
\hline$\omega_{b}$ & $68 \%$ & $0.0229(0.0223,0.0236)$ & $0.0237(0.0230,0.0244)$ & $0.0230(0.0225,0.0235)$ & $0.0225(0.0221,0.0230)$ & $0.0231(0.0225,0.0237)$ \\
\hline$\omega_{c}$ & $68 \%$ & $0.1052(0.1002,0.1105)$ & $0.1062(0.1007,0.1113)$ & $0.1079(0.1030,0.1126)$ & $0.1162(0.1113,0.1212)$ & $0.1093(0.1040,0.1146)$ \\
\hline $100 \theta$ & $68 \%$ & $1.0472(1.0435,1.0505)$ & $1.0436(1.0415,1.0459)$ & $1.0438(1.0415,1.0462)$ & $1.0391(1.0370,1.0412)$ & $1.0419(1.0398,1.0440)$ \\
\hline$\tau$ & $68 \%$ & $0.0906(0.0772,0.1046)$ & $0.0922(0.0790,0.1067)$ & $0.0866(0.0741,0.1007)$ & $0.0903(0.0764,0.1046)$ & $0.0899(0.0757,0.1045)$ \\
\hline$\Omega_{\Lambda}$ & $68 \%$ & $0.7798(0.7526,0.8029)$ & $0.7700(0.7430,0.7976)$ & $0.7611(0.7366,0.7849)$ & $0.7076(0.6768,0.7342)$ & $0.7508(0.7221,0.7769)$ \\
\hline$H_{0}$ & $68 \%$ & $76.24(73.26,79.26)$ & $75.16(72.40,78.60)$ & $74.02(71.59,76.67)$ & $68.85(66.59,71.17)$ & $72.95(70.27,75.69)$ \\
\hline $\ln \left[10^{10} A_{0}^{2}\right]$ & $68 \%$ & $3.0931(3.0540,3.1404)$ & $3.1725(3.1211,3.2281)$ & $3.0859(3.0498,3.1223)$ & $3.1689(3.1316,3.2074)$ & $3.1038(3.0641,3.1422)$ \\
\hline$n_{\mathrm{ad}}^{\mathrm{eff}}$ & $68 \%$ & $0.9961(0.9788,1.0149)$ & $1.0030(0.9839,1.0261)$ & $0.9902(0.9751,1.0064)$ & $0.9575(0.9430,0.9712)$ & $0.9859(0.9685,1.0041)$ \\
\hline$\gamma_{1}$ & $95 \%$ & $>-0.1275$ & & & & \\
\hline$\gamma_{2}$ & $95 \%$ & $>-0.1042$ & & & & \\
\hline$\alpha_{1}$ & $95 \%$ & $<0.0454$ & $<0.1114$ & $<0.0148$ & $<0.0073$ & \\
\hline$\alpha_{2}$ & $95 \%$ & $<0.3815$ & $<0.5012$ & $<0.0148$ & $<0.0073$ & \\
\hline$\tilde{r}_{0}$ & $95 \%$ & $<0.5238$ & $<0.2925$ & & & $<0.3334$ \\
\hline$\gamma_{0}$ & $95 \%$ & $>-0.1126$ & & & & \\
\hline$\alpha_{0}$ & $95 \%$ & $<0.0955$ & $<0.2110$ & $<0.0148$ & $<0.0073$ & \\
\hline$n_{\mathrm{ar}}$ & $95 \%$ & $0.9964(0.8057,1.1627)$ & $1.0030(0.9673,1.0506)$ & & & $0.9859(0.9545,1.0236)$ \\
\hline$n_{\text {as }}$ & $95 \%$ & $0.9995(0.6086,1.3376)$ & & $0.9902(0.9611,1.0218)$ & $0.9575(0.9284,0.9845)$ & \\
\hline$n_{\text {iso }}$ & $95 \%$ & $2.0523(0.8075,3.3592)$ & $1.7338(0.9795,2.7873)$ & $0.9902(0.9611,1.0218)$ & $0.9575(0.9284,0.9845)$ & \\
\hline$r_{0}$ & $95 \%$ & $<0.2571$ & $<0.2925$ & & & $<0.3334$ \\
\hline$\alpha_{\text {cor } 0}$ & $95 \%$ & $0.0747(-0.0770,0.1499)$ & & $0.0531(0.0100,0.1206)$ & $-0.0368(-0.0852,-0.0063)$ & \\
\hline$\alpha_{T}$ & $95 \%$ & $0.0209(-0.0295,0.0467)$ & $<0.0358$ & $0.0272(0.0055,0.0582)$ & $-0.0214(-0.0532,-0.0035)$ & \\
\hline$-\ln Z$ & & 3905.93 & 3902.82 & 3904.71 & 3905.49 & 3901.17 \\
\hline $\ln \left(Z_{\text {adiab }} / Z\right)$ & & 4.8 & 1.7 & 3.5 & 4.3 & 0 \\
\hline$Z_{\text {adiab }} / Z$ & & 117 & 5.2 & 34 & 75 & 1 \\
\hline
\end{tabular}


TABLE V. Slow-roll parametrization, NDI. The median values and $68 \%$ or $95 \%$ C.L. intervals (in parentheses) are given for a selection of parameters. For some parameters the whole prior range (p.r.) is allowed by the data. Note: $\gamma= \pm 1$ models do not have a tensor contribution (since $r_{0}=0$ automatically), and the only "slow-roll parameter" is then the combination $\eta_{s s}-\varepsilon$ for which we assume a uniform prior $(-0.075,0.075)$.

\begin{tabular}{|c|c|c|c|c|c|c|}
\hline Parameter & C.L. & Mixed NDI, gen. corr. & Mixed NDI, $\gamma=0$ & Mixed NDI, $\gamma=1$ & Mixed NDI, $\gamma=-1$ & Adiabatic \\
\hline$\omega_{b}$ & $68 \%$ & $0.0234(0.0229,0.0240)$ & $0.0236(0.0229,0.0243)$ & $0.0233(0.0228,0.0239)$ & $0.0224(0.0219,0.0229)$ & $0.0231(0.0226,0.0236)$ \\
\hline$\omega_{c}$ & $68 \%$ & $0.1077(0.1030,0.1122)$ & $0.1064(0.1010,0.1114)$ & $0.1092(0.1046,0.1136)$ & $0.1145(0.1102,0.1190)$ & $0.1095(0.1045,0.1142)$ \\
\hline$\theta$ & $68 \%$ & $1.0454(1.0428,1.0484)$ & $1.0437(1.0413,1.0464)$ & $1.0457(1.0431,1.0486)$ & $1.0390(1.0365,1.0411)$ & $1.0418(1.0400,1.0437)$ \\
\hline$\tau$ & $68 \%$ & $0.0876(0.0752,0.1014)$ & $0.0901(0.0768,0.1041)$ & $0.0884(0.0749,0.1014)$ & $0.0891(0.0755,0.1025)$ & $0.0899(0.0763,0.1034)$ \\
\hline $\ln \left[10^{10} A_{0}^{2}\right]$ & $68 \%$ & $3.0686(3.0312,3.1081)$ & $3.1739(3.1167,3.2499)$ & $3.0710(3.0355,3.1098)$ & $3.1663(3.1307,3.2057)$ & $3.1059(3.0690,3.1409)$ \\
\hline$\Omega_{\Lambda}$ & $68 \%$ & $0.7669(0.7427,0.7905)$ & $0.7699(0.7427,0.7966)$ & $0.7611(0.7364,0.7847)$ & $0.7151(0.6865,0.7397)$ & $0.7495(0.7238,0.7750)$ \\
\hline$H_{0}$ & $68 \%$ & $75.02(72.46,77.89)$ & $75.13(72.19,78.48)$ & $74.47(71.84,77.26)$ & $69.33(67.06,71.53)$ & $72.75(70.40,75.36)$ \\
\hline$n_{\mathrm{ad}}^{\text {eff }}$ & $68 \%$ & $0.9879(0.9742,1.0026)$ & $0.9982(0.9805,1.0178)$ & $0.9853(0.9720,0.9990)$ & $0.9639(0.9509,0.9766)$ & $0.9843(0.9697,1.0021)$ \\
\hline$\gamma_{0}$ & $95 \%$ & $>-0.3178$ & & & & \\
\hline$\alpha_{0}$ & $95 \%$ & $<0.0568$ & $<0.2482$ & $<0.0280$ & $<0.0104$ & \\
\hline$\eta_{\sigma \sigma}$ & $95 \%$ & p.r. & $0.0146(-0.0129,0.0641)$ & & & $0.0102(-0.0175,0.0637)$ \\
\hline$\eta_{\sigma s}$ & $95 \%$ & p.r. & & & & \\
\hline$\eta_{s s}$ & $95 \%$ & p.r. & p.r. & & & \\
\hline$\varepsilon$ & $95 \%$ & $<0.0570$ & $<0.0152$ & & & $<0.0193$ \\
\hline$\eta_{s s}-\varepsilon$ & $95 \%$ & & & $-0.0073(-0.0202,0.0061)$ & $-0.0180(-0.0311,-0.0056)$ & \\
\hline$n_{\mathrm{ar}}$ & $95 \%$ & $0.9486(0.6564,1.0805)$ & $0.9982(0.9649,1.0400)$ & & & $0.9843(0.9561,1.0183)$ \\
\hline$n_{\mathrm{as}}$ & $95 \%$ & $0.9981(0.8459,1.1719)$ & & $0.9853(0.9595,1.0122)$ & $0.9639(0.9378,0.9888)$ & \\
\hline$n_{\text {iso }}$ & $95 \%$ & $0.9957(0.8548,1.1206)$ & $1.0129(0.8475,1.1325)$ & $0.9853(0.9595,1.0122)$ & $0.9639(0.9378,0.9888)$ & \\
\hline$r_{0}$ & $95 \%$ & $<0.1909$ & $<0.2439$ & & & $<0.3083$ \\
\hline$\alpha_{\text {cor0 } 0}$ & $95 \%$ & $0.0729(-0.0424,0.1535)$ & & $0.0848(0.0194,0.1649)$ & $-0.0378(-0.1015,-0.0061)$ & \\
\hline$\alpha_{T}$ & $95 \%$ & $0.0405(-0.0222,0.0840)$ & $<0.0503$ & $0.0463(0.0110,0.0881)$ & $-0.0222(-0.0623,-0.0035)$ & \\
\hline$-\ln Z$ & & 3901.52 & 3900.19 & 3901.09 & 3903.60 & 3898.94 \\
\hline $\ln \left(Z_{\text {adiab }} / Z\right)$ & & 2.6 & 1.3 & 2.2 & 4.7 & 0 \\
\hline$Z_{\text {adiab }} / Z$ & & 13 & 3.5 & 8.6 & 106 & 1 \\
\hline
\end{tabular}

TABLE VI. Slow-roll paramerization, CDI. The median values and 68\% or 95\% C.L. intervals (in parentheses) are given for a selection of parameters. For some parameters the whole prior range (p.r.) is allowed by the data. Note: $\gamma= \pm 1$ models do not have a tensor contribution (since $r_{0}=0$ automatically), and the only "slow-roll parameter" is then the combination $\eta_{s s}-\varepsilon$ for which we assume a uniform prior $(-0.075,0.075)$.

\begin{tabular}{|c|c|c|c|c|c|c|}
\hline Parameter & C.L. & Mixed CDI, gen. corr. & Mixed CDI, $\gamma=0$ & Mixed CDI, $\gamma=1$ & Mixed CDI, $\gamma=-1$ & Adiabatic \\
\hline$\omega_{b}$ & $68 \%$ & $0.0231(0.0226,0.0236)$ & $0.0234(0.0228,0.0241)$ & $0.0230(0.0225,0.0235)$ & $0.0225(0.0220,0.0230)$ & $0.0231(0.0226,0.0236)$ \\
\hline$\omega_{c}$ & $68 \%$ & $0.1088(0.1029,0.1150)$ & $0.1068(0.1018,0.1119)$ & $0.1077(0.1029,0.1129)$ & $0.1164(0.1113,0.1215)$ & $0.1095(0.1045,0.1142)$ \\
\hline$\theta$ & $68 \%$ & $1.0421(1.0390,1.0452)$ & $1.0426(1.0405,1.0447)$ & $1.0440(1.0417,1.0464)$ & $1.0390(1.0368,1.0413)$ & $1.0418(1.0400,1.0437)$ \\
\hline$\tau$ & $68 \%$ & $0.0894(0.0763,0.1030)$ & $0.0894(0.0757,0.1037)$ & $0.0862(0.0733,0.1003)$ & $0.0904(0.0757,0.1059)$ & $0.0899(0.0763,0.1034)$ \\
\hline $\ln \left[10^{10} A_{0}^{2}\right]$ & $68 \%$ & $3.1042(3.0558,3.1660)$ & $3.1207(3.0812,3.1595)$ & $3.0836(3.0469,3.1227)$ & $3.1706(3.1333,3.2105)$ & $3.1059(3.0690,3.1409)$ \\
\hline$\Omega_{\Lambda}$ & $68 \%$ & $0.7539(0.7162,0.7851)$ & $0.7650(0.7379,0.7896)$ & $0.7620(0.7360,0.7858)$ & $0.7062(0.6725,0.7356)$ & $0.7495(0.7238,0.7750)$ \\
\hline$H_{0}$ & $68 \%$ & $73.15(69.62,76.75)$ & $74.43(71.72,77.31)$ & $74.09(71.62,76.91)$ & $68.78(66.23,71.27)$ & $72.75(70.40,75.36)$ \\
\hline$n_{\text {ad }}^{\text {eff }}$ & $68 \%$ & $0.9848(0.9623,1.0042)$ & $0.9973(0.9796,1.0168)$ & $0.9905(0.9757,1.0071)$ & $0.9565(0.9412,0.9716)$ & $0.9843(0.9697,1.0021)$ \\
\hline$\gamma_{0}$ & $95 \%$ & p.r. & & & & \\
\hline$\alpha_{0}$ & $95 \%$ & $<0.0298$ & $<0.1171$ & $<0.0125$ & $<0.0064$ & \\
\hline$\eta_{\sigma \sigma}$ & $95 \%$ & p.r. & $0.0154(-0.0134,0.0628)$ & & & $0.0102(-0.0175,0.0637)$ \\
\hline$\eta_{\sigma s}$ & $95 \%$ & p.r. & & & & \\
\hline$\eta_{s s}$ & $95 \%$ & p.r. & p.r. & & & \\
\hline$\varepsilon$ & $95 \%$ & $<0.0378$ & $<0.0157$ & & & $<0.0193$ \\
\hline$\eta_{s s}-\varepsilon$ & $95 \%$ & & & $-0.0048(-0.0193,0.0113)$ & $-0.2176(-0.0369,-0.0068)$ & \\
\hline$n_{\mathrm{ar}}$ & $95 \%$ & $0.9657(0.7220,1.0712)$ & $0.9973(0.9636,1.0370)$ & & & $0.9843(0.9561,1.0183)$ \\
\hline$n_{\text {as }}$ & $95 \%$ & $0.9938(0.6802,1.1844)$ & & $0.9905(0.9613,1.0226)$ & $0.9565(0.9263,0.9863)$ & \\
\hline$n_{\text {iso }}$ & $95 \%$ & $0.9889(0.8459,1.1219)$ & $1.0199(0.8481,1.1355)$ & $0.9905(0.9613,1.0226)$ & $0.9565(0.9263,0.9863)$ & \\
\hline$r_{0}$ & $95 \%$ & $<0.2033$ & $<0.2516$ & & & $<0.3083$ \\
\hline$\alpha_{\text {cor } 0}$ & $95 \%$ & $0.0167(-0.0836,0.1004)$ & & $0.0542(0.0113,0.1249)$ & $-0.0379(-0.0871,-0.0076)$ & \\
\hline$\alpha_{T}$ & $95 \%$ & $0.0093(-0.0448,0.0497)$ & $<0.0404$ & $0.3607(0.0083,0.0541)$ & $-0.0219(-0.0550,-0.0043)$ & \\
\hline$-\ln Z$ & & 3902.87 & 3901.74 & 3902.40 & 3903.86 & 3898.94 \\
\hline $\ln \left(Z_{\text {adiab }} / Z\right)$ & & 3.9 & 2.8 & 3.5 & 4.9 & 0 \\
\hline$Z_{\text {adiab }} / Z$ & & 51 & 16 & 33 & 137 & 1 \\
\hline
\end{tabular}


[1] J. Valiviita, M. Savelainen, M. Talvitie, H. Kurki-Suonio, and S. Rusak, Astrophys. J. 753, 151 (2012).

[2] C. Bennett et al. (WMAP Collaboration), arXiv:1212.5225.

[3] D. Larson, J. Dunkley, G. Hinshaw, E. Komatsu, M. Nolta et al., Astrophys. J. Suppl. Ser. 192, 16 (2011).

[4] C. L. Reichardt et al., Astrophys. J. 694, 1200 (2009).

[5] M. Brown et al. (QUaD collaboration), Astrophys. J. 705, 978 (2009).

[6] P. Ade et al. (Planck Collaboration), arXiv:1303.5082.

[7] M. Bucher, K. Moodley, and N. Turok, Phys. Rev. D 62, 083508 (2000).

[8] D. Grin, D. Hanson, G. Holder, O. Doré, and M. Kamionkowsk, arXiv:1306.4319.

[9] D. Grin, O. Doré, and M. Kamionkowski, Phys. Rev. D 84, 123003 (2011).

[10] A. Lewis, http://cosmologist.info/notes/CAMB.pdf.

[11] D. Langlois and B. van Tent, J. Cosmol. Astropart. Phys. 07 (2012) 040.

[12] K. Enqvist, H. Kurki-Suonio, and J. Valiviita, Phys. Rev. D 62, 103003 (2000).

[13] K. Enqvist, H. Kurki-Suonio, and J. Valiviita, Phys. Rev. D 65, 043002 (2002).

[14] M. Beltran, J. Garcia-Bellido, J. Lesgourgues, A. R. Liddle, and A. Slosar, Phys. Rev. D 71, 063532 (2005).

[15] M. Beltran, J. Garcia-Bellido, J. Lesgourgues, and M. Viel, Phys. Rev. D 72, 103515 (2005).

[16] R. Keskitalo, H. Kurki-Suonio, V. Muhonen, and J. Valiviita, J. Cosmol. Astropart. Phys. 09 (2007) 008.

[17] R. Trotta, Mon. Not. R. Astron. Soc. Lett. 375, L26 (2007).

[18] U. Seljak, A. Slosar, and P. McDonald, J. Cosmol. Astropart. Phys. 10 (2006) 014.

[19] A. Lewis, arXiv:astro-ph/0603753.

[20] R. Bean, J. Dunkley, and E. Pierpaoli, Phys. Rev. D 74, 063503 (2006).

[21] M. Kawasaki and T. Sekiguchi, Prog. Theor. Phys. 120, 995 (2008).

[22] M. Beltran, Phys. Rev. D 78, 023530 (2008).

[23] I. Sollom, A. Challinor, and M. P. Hobson, Phys. Rev. D 79, 123521 (2009).

[24] J. Valiviita and T. Giannantonio, Phys. Rev. D 80, 123516 (2009).

[25] P. Castro et al. (QUaD Collaboration), Astrophys. J. 701, 857 (2009).

[26] H. Li, J. Liu, J.-Q. Xia, and Y.-F. Cai, Phys. Rev. D 83, 123517 (2011).

[27] H. Peiris et al. (WMAP Collaboration), Astrophys. J. Suppl. Ser. 148, 213 (2003).

[28] J. Valiviita and V. Muhonen, Phys. Rev. Lett. 91, 131302 (2003).

[29] H. Kurki-Suonio, V. Muhonen, and J. Valiviita, Phys. Rev. D 71, 063005 (2005).

[30] J. E. Lidsey, A. R. Liddle, E. W. Kolb, E. J. Copeland, T. Barreiro, and M. Abney, Rev. Mod. Phys. 69, 373 (1997).

[31] N. Bartolo, S. Matarrese, and A. Riotto, Phys. Rev. D 64, 123504 (2001).

[32] D. Wands, N. Bartolo, S. Matarrese, and A. Riotto, Phys. Rev. D 66, 043520 (2002).

[33] M. Cortes and A.R. Liddle, Phys. Rev. D 73, 083523 (2006).
[34] M. Cortes, A. R. Liddle, and P. Mukherjee, Phys. Rev. D 75, 083520 (2007).

[35] C. T. Byrnes and D. Wands, Phys. Rev. D 74, 043529 (2006).

[36] B. van Tent, Classical Quantum Gravity 21, 349 (2004).

[37] C. M. Peterson and M. Tegmark, Phys. Rev. D 83, 023522 (2011).

[38] J. Norena, C. Wagner, L. Verde, H. V. Peiris, and R. Easther, Phys. Rev. D 86, 023505 (2012).

[39] K.-Y. Choi, J.-O. Gong, and D. Jeong, J. Cosmol. Astropart. Phys. 02 (2009) 032.

[40] T. Hamazaki, Nucl. Phys. B791, 20 (2008).

[41] F. Di Marco, F. Finelli, and A. Gruppuso, Phys. Rev. D 76, 043530 (2007).

[42] Z. Lalak, D. Langlois, S. Pokorski, and K. Turzynski, J. Cosmol. Astropart. Phys. 07 (2007) 014.

[43] K.-Y. Choi, L. M. H. Hall, and C. van de Bruck, J. Cosmol. Astropart. Phys. 02 (2007) 029.

[44] G. I. Rigopoulos, E. P. S. Shellard, and B. J. W. van Tent, Phys. Rev. D 76, 083512 (2007).

[45] B. A. Bassett, S. Tsujikawa, and D. Wands, Rev. Mod. Phys. 78, 537 (2006).

[46] T. Hattori and K. Yamamoto, J. Cosmol. Astropart. Phys. 07 (2005) 005.

[47] F. Di Marco and F. Finelli, Phys. Rev. D 71, 123502 (2005).

[48] D. Parkinson, S. Tsujikawa, B.A. Bassett, and L. Amendola, Phys. Rev. D 71, 063524 (2005).

[49] A. Gruzinov, arXiv:astro-ph/0401407.

[50] N. Bartolo, P.S. Corasaniti, A. R. Liddle, and M. Malquarti, Phys. Rev. D 70, 043532 (2004).

[51] F. Vernizzi, Phys. Rev. D 69, 083526 (2004).

[52] W. Lee and L.-Z. Fang, Phys. Rev. D 69, 023514 (2004).

[53] A. Mazumdar and M. Postma, Phys. Lett. B 573, 5 (2003).

[54] K. A. Malik, D. Wands, and C. Ungarelli, Phys. Rev. D 67, 063516 (2003).

[55] F. Di Marco, F. Finelli, and R. Brandenberger, Phys. Rev. D 67, 063512 (2003).

[56] P. R. Ashcroft, C. van de Bruck, and A.C. Davis, Phys. Rev. D 69, 083516 (2004).

[57] F. Bernardeau and J.-P. Uzan, Phys. Rev. D 66, 103506 (2002).

[58] S. Tsujikawa and B. A. Bassett, Phys. Lett. B 536, 9 (2002).

[59] A. A. Starobinsky, S. Tsujikawa, and J. Yokoyama, Nucl. Phys. B610, 383 (2001).

[60] S. Groot Nibbelink and B. J.W. van Tent, Classical Quantum Gravity 19, 613 (2002).

[61] N. Bartolo, S. Matarrese, and A. Riotto, Phys. Rev. D 64, 083514 (2001).

[62] J.-c. Hwang and H. Noh, Classical Quantum Gravity 19, 527 (2002).

[63] J.-c. Hwang and H. Noh, Phys. Lett. B 495, 277 (2000).

[64] C. Gordon, D. Wands, B. A. Bassett, and R. Maartens, Phys. Rev. D 63, 023506 (2000).

[65] A. N. Taylor and A. Berera, Phys. Rev. D 62, 083517 (2000).

[66] F. Finelli and R.H. Brandenberger, Phys. Rev. D 62, 083502 (2000).

[67] A. R. Liddle and A. Mazumdar, Phys. Rev. D 61, 123507 (2000). 
[68] B. A. Bassett, C. Gordon, R. Maartens, and D. I. Kaiser, Phys. Rev. D 61, 061302 (2000).

[69] E. Pierpaoli, J. Garcia-Bellido, and S. Borgani, J. High Energy Phys. 10 (1999) 015.

[70] D. Langlois, Phys. Rev. D 59, 123512 (1999).

[71] G. N. Felder, L. Kofman, and A. D. Linde, Phys. Rev. D 60, 103505 (1999).

[72] F. Perrotta and C. Baccigalupi, Phys. Rev. D 59, 123508 (1999).

[73] T. Chiba, N. Sugiyama, and J. Yokoyama, Nucl. Phys. B530, 304 (1998).

[74] T. T. Nakamura and E. D. Stewart, Phys. Lett. B 381, 413 (1996).

[75] D. Polarski and A. A. Starobinsky, Phys. Rev. D 50, 6123 (1994).

[76] D. H. Lyth, C. Ungarelli, and D. Wands, Phys. Rev. D 67, 023503 (2003).

[77] C. Gordon and K. A. Malik, Phys. Rev. D 69, 063508 (2004).

[78] M. Kawasaki, K. Miyamoto, K. Nakayama, and T. Sekiguchi, J. Cosmol. Astropart. Phys. 02 (2012) 022.

[79] E. Di Valentino, M. Lattanzi, G. Mangano, A. Melchiorri, and P. Serpico, Phys. Rev. D 85, 043511 (2012).

[80] M. Orito, T. Kajino, G. Mathews, and Y. Wang, Phys. Rev. D 65, 123504 (2002).

[81] P. Ade et al. (Planck Collaboration), arXiv:1303.5084.
[82] F. Feroz, M. Hobson, and M. Bridges, Mon. Not. R. Astron. Soc. 398, 1601 (2009).

[83] http://ccpforge.cse.rl.ac.uk/gf/project/multinest/.

[84] J. Skilling, AIP Conf. Proc. 735, 395 (2004).

[85] P. Mukherjee, D. Parkinson, and A. R. Liddle, Astrophys. J. 638, L51 (2006).

[86] R. Shaw, M. Bridges, and M. P. Hobson, Mon. Not. R. Astron. Soc. 378, 1365 (2007).

[87] F. Feroz and M. Hobson, Mon. Not. R. Astron. Soc. 384, 449 (2008).

[88] A. Lewis, A. Challinor, and A. Lasenby, Astrophys. J. 538, 473 (2000).

[89] A. Lewis and S. Bridle, Phys. Rev. D 66, 103511 (2002).

[90] M. Bucher, K. Moodley, and N. Turok, Phys. Rev. D 66, 023528 (2002).

[91] M. Bucher, K. Moodley, and N. Turok, Phys. Rev. Lett. 87, 191301 (2001).

[92] S. M. Kasanda, C. Zunckel, K. Moodley, B. Bassett, and P. Okouma, J. Cosmol. Astropart. Phys. 07 (2012) 021.

[93] C. Zunckel, P. Okouma, S. Kasanda, K. Moodley, and B. Bassett, Phys. Lett. B 696, 433 (2011).

[94] R. Laureijs et al. (EUCLID Collaboration), arXiv:1110.3193.

[95] C. Carbone, A. Mangilli, and L. Verde, J. Cosmol. Astropart. Phys. 09 (2011) 028. 